\title{
COVARIANCE STABILITY AND THE 2008 FINANCIAL CRISIS: THE IMPACT IN THE PORTFOLIO OF THE 10 BIGGEST COMPANIES IN BM\&FBOVESPA BETWEEN 2004 E 2012
}

\author{
Leticia Naomi Ono Maeda \\ UNICAMP - Universidade Estadual de Campinas, Brazil \\ E-mail: leticiamaeda@gmail.com \\ Johan Hendrik Poker \\ UNICAMP - Universidade Estadual de Campinas, Brazil \\ E-mail: johan.poker@fca.unicamp.br
}

Submission: 15/10/2015

Revision: 08/11/2015

\section{ABSTRACT}

Accept: 20/11/2015

This study's purpose is to analyze the influence of the covariance fluctuation between assets over the structure of a portfolio of investments. To accomplish that, the covariances between the daily returns of the 10 biggest participants of the BM\&FBovespa stock market are analyzed, before, during and after the 2008 financial crisis. The procedure of this research includes: (1) collection of returns of the selected stocks between 2004 and 2012; (2) composition of the classical portfolio proposed by Markowitz's theory (1952); and (3) the measurement of the covariances instability effect between the 10 selected assets over the maintenance of a portfolio's risk and return, according to the preferences of a hypothetical investor. We discover that the asset's covariance vary over time and affect the correlations among the assets, especially in financial crisis periods. Consequently, both risk and return of the portfolio may change greatly if the asset's weights are not recalculated periodically. This supports the idea that portfolio theory might benefit from the development of stability weighted techniques.

Keywords: portfolio theory; asset risk management; financial crisis. 
DOI: 10.14807/ijmp.v7i1.324

\section{INTRODUCTION}

The ability to predict the future value of assets in the financial market was always desirable, and there are currently many ways to choose assets which compose a given investment portfolio, evaluating the assets characteristics such as their expected return, risk, investment period, liquidity, among others. One of the possible financial instruments that analyze the relationship between two of these characteristics - precisely, risk and return -to elect the best investment option is the Markowitz model (1952).

However, it is crucial to clarify that the covariance stability between the companies is assumed over time, so that the chosen investment portfolio according to the Markowitz model (1952) is maintained during the investment period. Thus, if the covariances are unstable, possible commitments related to the expected portfolios results may occur.

Since covariances are dynamic and dependent on economy variations in general and, specifically, on the financial market, this study is justified by the need to assess to what extent and in what kind of scenario it would be unwise to use the Markowitz model (1952) - especially in economic instability situations, as in the recent 2008 crisis - with no use of improvements, in order not to put at risk results expected from a portfolio.

\section{THEORETICAL FOUNDATION}

\subsection{Markowitz model (1952)}

According to the Markowitz model (1952), an investor tries to predict the future outcome of assets basically through the analysis of expected return and risk of the asset. The latter, in turn, is considered, according to Luenberg (1997), as random variables, since the asset can take different future values, each with a given probability of occurrence, considering that the future asset value is not known upon purchase.

Thus, mathematically speaking, the expected return is basically the sum of the possible asset returns ${ }^{x_{i}}$ weighted by their probabilities ${ }^{p_{i}}$ of occurence, whereas the risk is in the variance - or on the square of the variance (standard deviation) which is 
DOI: 10.14807/ijmp.v7i1.324

most routinely used - of the aforesaid return, that is, the calculation of how far a value is from its expected value. Both described in the following formulas,

$$
\bar{x}=\sum_{i=1}^{m} x_{i} p_{\bar{i}}
$$

Where

$-\bar{x}=$ expected value of asset $\mathrm{X}$;

$-x_{i}=$ value of asset $\mathrm{X}$ in time $\mathrm{i}$;

$-p_{\bar{i}}=$ probability of the value of asset $\mathrm{X}$ in time $\mathrm{i}$.

$$
\sigma_{x}=\sqrt{E\left[\left(x_{i}-\bar{x}\right)^{2}\right] p_{i}}
$$

Where

- $\sigma_{x}=$ standard deviation of the asset X;

$-x_{i}=$ value of asset $\mathrm{X}$ in the time $\mathrm{i}$;

$-\bar{x}=$ expected value of the asset $\mathrm{X}$;

$-p_{i}=$ probability of the value of asset $\mathrm{X}$ in the time $\mathrm{i}$.

Usually, however, one does not invest in a single asset but in a set of assets, named assets portfolio or investment portfolio. The preference for an investment portfolio to only one asset occurs due to the need to diminish the risk of an investment. According to Bodie et al., (2010), the risk may be classified as nondiversifiable risk and diversifiable risk. The first is the risk inherent in the market as a whole, whereas the second is closely related to one or more specific parts of the market and, therefore, may be minimized by diversifying assets, that is, an investment of a specific amount in different assets of the financial market. Markowitz (1952, p. 89) describes this phenomenon as follows:

\footnotetext{
"In an attempt to reduce variance, investing in various assets is not enough. One must avoid that the investment is made in assets with high covariance between them. We must diversify investment among industries, particularly industries with different economic characteristics, since companies from different industries have lower covariance than companies in the same industry."
} 
DOI: 10.14807/ijmp.v7i1.324

In this respect, composing an assets portfolio decreases diversifiable risk significantly, increasing the probability of an asset to obtain a certain expected value, or in other words, reducing risk. However, we still need to understand how we should select some on them among the various assets available in the market, which can form what Markowitz (1952) named as efficient portfolio investment. A portfolio is effective for a given return, there is no other portfolio with less risk, or, similarly, for a given risk, there is no other portfolio with a higher return. This concept can also be interpreted by the Sharpe Dominance Principle (1965):

"An investor should choose their optimal portfolio from the set of portfolios that:

1. Offers maximum expected return for different levels of risk, and

2. Offers minimal risk for different levels of expected return."

Thus, in order to calculate the expected return and the risk of a portfolio, it is assumed that an investor distributes an amount $X_{0}$ between $n$ assets, each with a weight $w_{i}$ in the portfolio, whereas $\sum_{i=1}^{n} W i=1$ and $X_{0}=\sum_{i=1}^{n} X_{i}$ where $X_{i}$ is the amount invested in the $i^{\text {th }}$ asset, it follows that the total return of the portfolio is given by:

$$
\mu_{p}=\sum_{i=1}^{n}=w_{i} \bar{x}_{i}
$$

Where:

$-\mu_{p}=$ portfolio total expected return;

$-w_{i}=$ weight of the asset $\mathrm{i}$;

$-\bar{x}_{k}=$ total expected return of the asset i.

In order to calculate a portfolio variance, the covariance and correlation concept is necessary. Both the covariance and the correlation can be clarified as the interdependence of two random variables. With respect to correlation, it follows that:

- If $\rho(x, y)=0$, then $X$ and $Y$ are uncorrelated;

- If $\rho(x, y)=1$, then $\mathrm{X}$ and $\mathrm{Y}$ are perfectly correlated;

- If $\rho(x, y)=-1$, then $\mathrm{X}$ and $\mathrm{Y}$ are negatively correlated. 
INDEPENDENT JOURNAL OF MANAGEMENT \& PRODUCTION (IJM\&P)

http://www.ijmp.jor.br

v. 7, n. 1, January - March 2016

ISSN: 2236-269X

DOI: 10.14807/ijmp.v7i1.324

Furthermore, the covariance between two $\mathrm{X}$ and $\mathrm{Y}$ assets can be mathematically defined by:

$$
\operatorname{Cov}_{x y}=\rho_{x, y} \sigma_{x} \sigma_{y}
$$

By knowing the covariance value between two variables it is possible to calculate the standard deviation (risk) of a portfolio of two assets, which is given by:

$$
\sigma_{p}=\sqrt{\left(w_{x}^{2} \sigma_{x}^{2}\right)+\left(w_{y}^{2} \sigma_{y}^{2}\right)+2 w_{x} w_{y} \operatorname{Cov}_{x y}}
$$

Where:

- $\sigma_{p}=$ portfolio standard deviation;

$-w_{x}=$ weight of the asset $\mathrm{X}$;

- $w_{y}=$ weight of the asset $Y$;

- $\sigma_{x}=$ standard deviation of asset X;

- $\sigma_{y}=$ standard deviation of asset $Y$

- $\operatorname{Cov}_{x y}=$ covariance between assets $\mathrm{X}$ and $\mathrm{Y}$.

However, if we wish to know the variance of a portfolio with more than two assets, we just need to use, according to Luenberger (1997), the formula:

$$
\sigma_{p}^{2}=\sum_{i, j=1}^{n} w_{i} w_{j} \operatorname{Cov}_{i j}
$$

Where:

- $\sigma_{p}^{2}=$ portfolio total variance;

$-w_{i}=$ weight of the asset $\mathrm{i}$;

$-w_{j}=$ weight of the asset $j$;

- $\operatorname{Cov}_{i j}=$ covariance between asset $\mathrm{i}$ and $\mathrm{j}$.

Thus, we can reject that the variance of the portfolio is calculated from the covariance between pairs of assets. Recalling that $\operatorname{Cov}_{i i}=\sigma_{i}^{2}$. 
INDEPENDENT JOURNAL OF MANAGEMENT \& PRODUCTION (IJM\&P)

http://www.ijmp.jor.br

v. 7, n. 1, January - March 2016

ISSN: 2236-269X

DOI: 10.14807/ijmp.v7i1.324

Using these return and portfolio risk concepts we can relate them to a chart whose abscissa corresponds to the risk and the orderly, to the expected return. The points of the chart correspond to an investment portfolio involving certain assets. The points corresponding to these investment portfolios form return-risk curves. In this curve are presented investment portfolios composed of the same assets, however with different ${ }^{w_{i}}$ weights for each portfolio (point) of the curve.

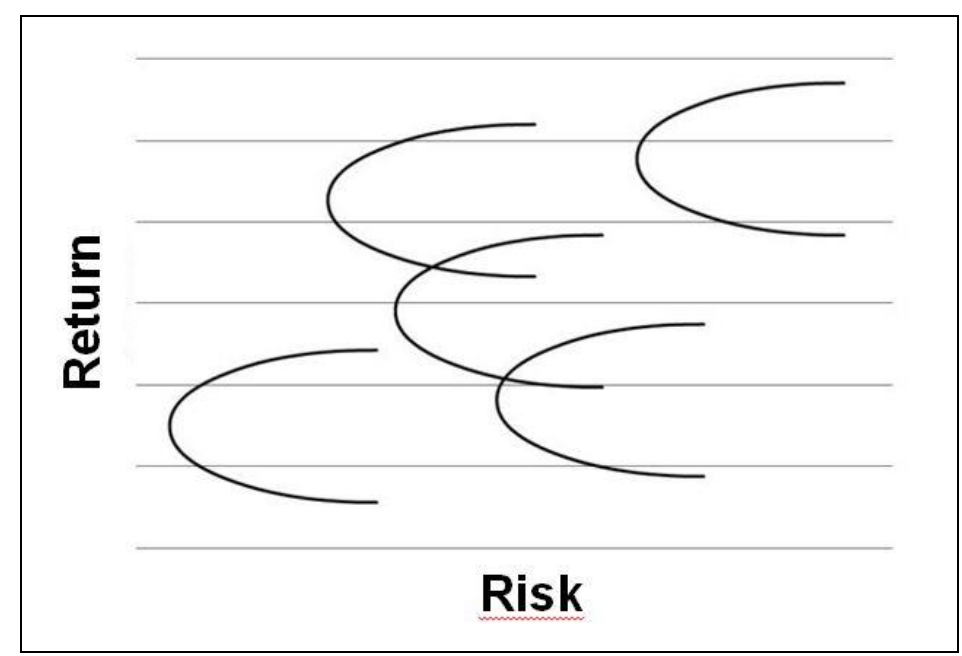

Figure 1: Return-Risk Curves.

Source: Adapted from Hieda and Oda (1998)

After choosing assets that will compose the portfolio, the corresponding RiskReturn curve to depreciated assets is found. Thus, we would obtain the following Risk-Return curve whose inner area is called feasible region. Both at the curved line as well as at the feasible region are all possible portfolios of the same assets, however with different ${ }^{w_{i}}$ weights.

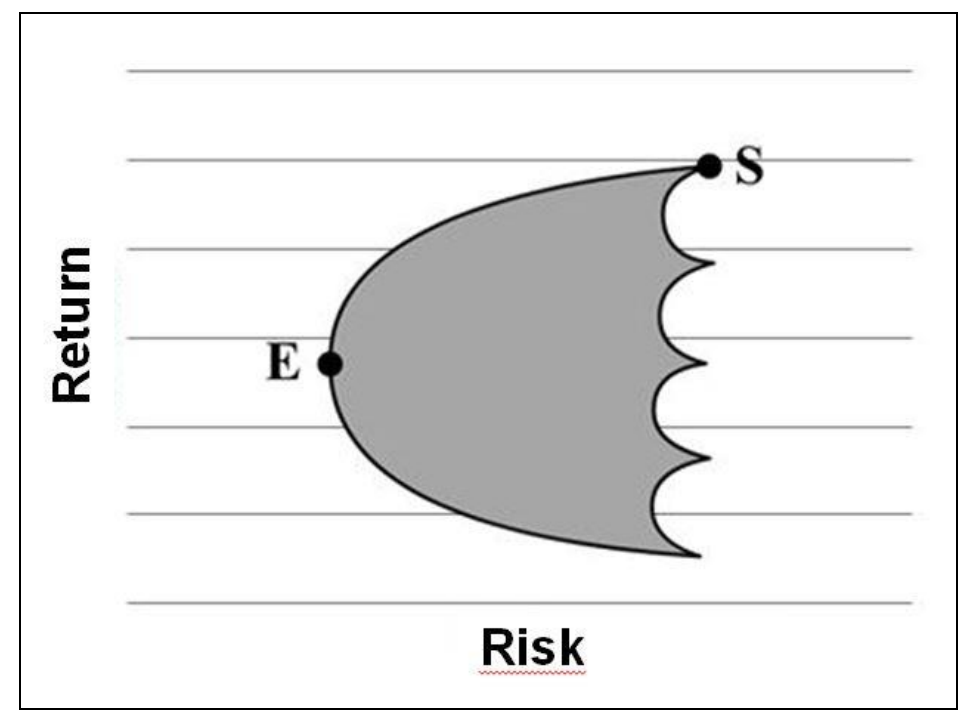

Figure 2: Feasible region

Source: Adapted from Luenberger (1997) 
INDEPENDENT JOURNAL OF MANAGEMENT \& PRODUCTION (IJM\&P)

http://www.ijmp.jor.br

v. 7, n. 1, January - March 2016

ISSN: 2236-269X

DOI: 10.14807/ijmp.v7i1.324

However the only part of the Risk-Return curve that follows the Dominance principle, cited above, corresponds to the curved line that goes from point "E" of minimal risk to point "S" of maximum return.

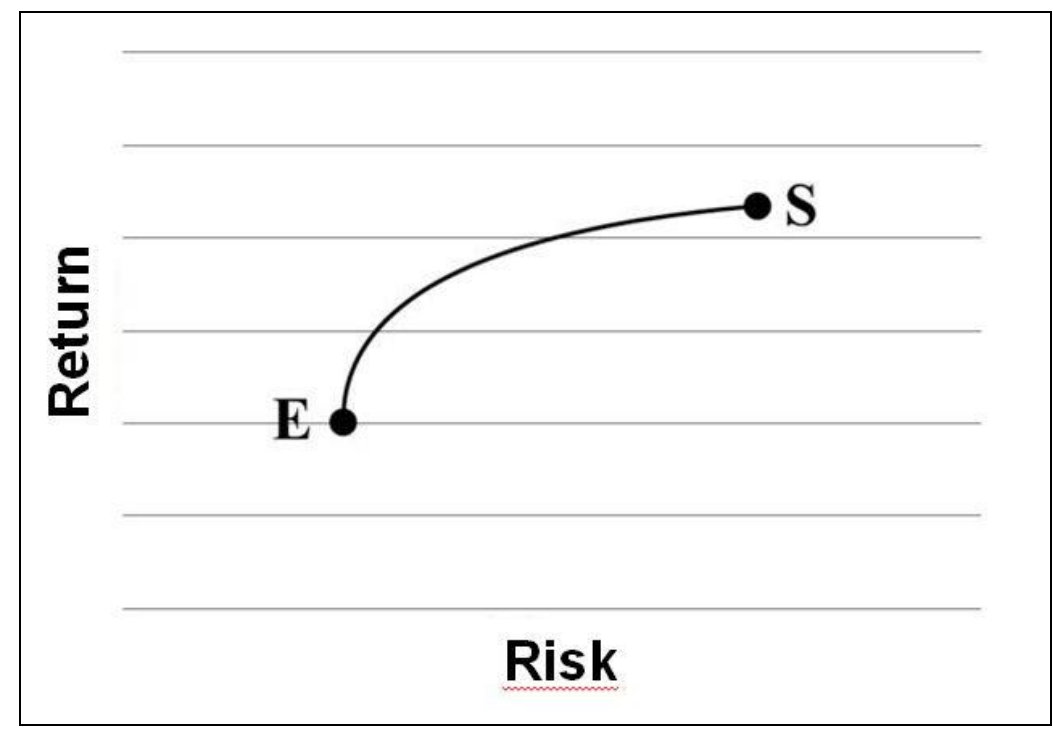

Figure 3: Efficient frontier

Source: Adapted from Hieda and Oda (1998)

The curve $\hat{E} S$ is called efficient frontier. Such boundary defines all the possible efficient portfolio investments, that is, those that for a given level of return have the minimum possible risk.

Finally, it is necessary to point out the relationship that the assets weights have with their correlation index. Assuming a portfolio composed of two $X$ and $Y$ assets, we form several $X$ and $Y$ combinations, each with a different correlation index among the same and different weights. Thus, short selling is not possible (weight of $X+$ weight of $Y=1$ ).

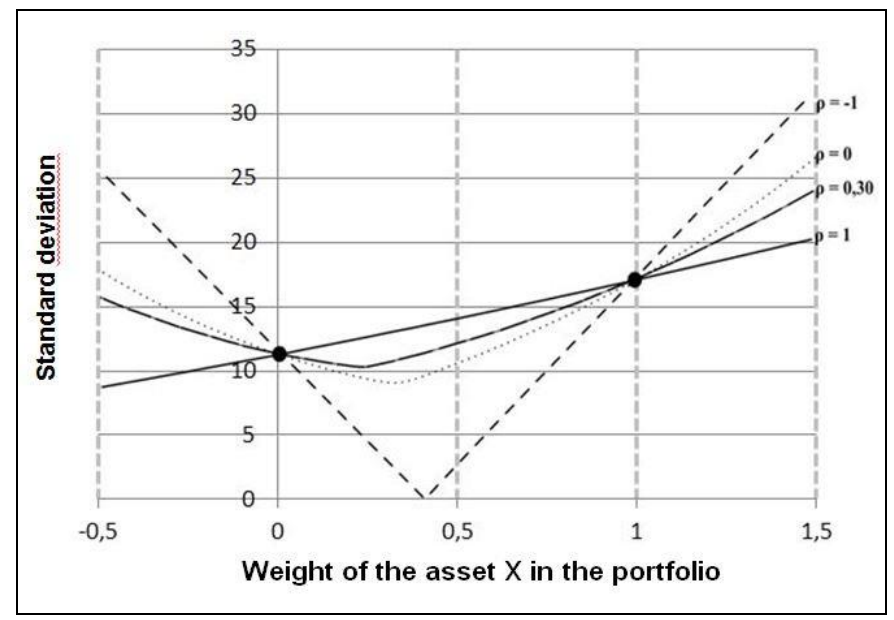

Chart 1: Correlation index

Source: Adapted from Bodie, Marcus and Kane (2010) 
INDEPENDENT JOURNAL OF MANAGEMENT \& PRODUCTION (IJM\&P)

http://www.ijmp.jor.br

v. 7, n. 1, January - March 2016

ISSN: 2236-269X

DOI: 10.14807/ijmp.v7i1.324

In it there is the following information on the assets correlation influence on the diversification effects:

- when the correlation between assets is positively perfect $(\rho=1)$, there is no diversification effect of assets;

- when the correlation between assets is imperfect $-1<\rho<1$, there are imperfect effects of asset diversification;

- when the correlation between assets is negatively perfect $(\rho=-1)$, there is a perfect effect of assets diversification shown by the scope of a risk equal to zero.

As the correlation between the two assets changes, the assets weights of the portfolio must also be changed in order to maintain a certain level of risk. For example, if it were necessary to maintain a minimum standard deviation, the asset $X$ should correspond to approximately $25 \%, 37.5 \%$ and $43.75 \%$ of the total portfolio, if the correlations were, respectively, $0,0.30$ and -1 - assets of correlation perfect in this case would not reach the aforementioned level of risk.

\subsection{Investor preferences}

Although the efficient frontier points the best investment combination alternatives, there is nothing on which combination or which portfolio should be selected, since this decision is up to each investor according to their personal characteristics.

According to Danthine (2005), such preferences may take into account several variables: the wealth degree of the investor, uncertainty in the investment time, among others. However, a good instrument for assessing the preference of an investor regarding the choice between risk assets is the indifference curve. 


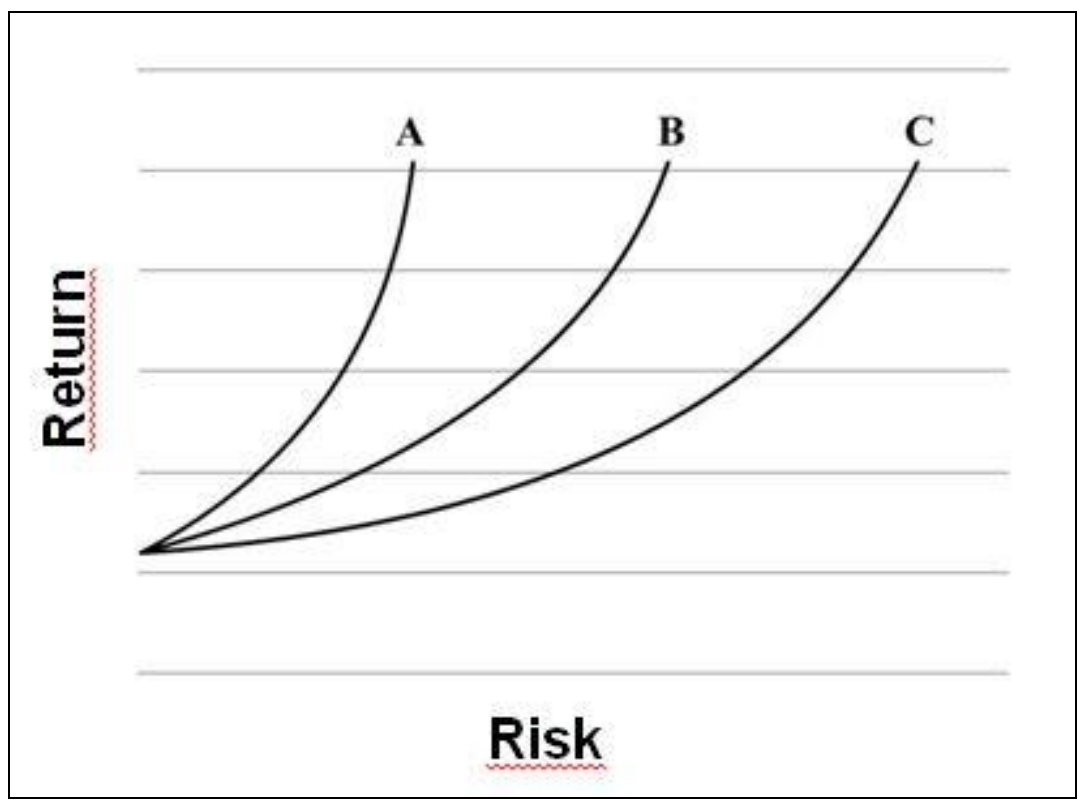

Figure 4: Risk aversion

Source: Adapted from Bodie, Marcus and Kane (2010)

The indifference curve measures the risk aversion degree of an investor, that is, the amount of additional return they need to accept one more risk unit. In the above, we observe three indifference curves. The steeper the curve, the greater the risk aversion degree is. Thus, the curves "A", "B" and "C" correspond to investors of, respectively, greater risk aversion, moderate risk aversion and lower risk aversion.

Similarly, risk aversion can also be calculated by the Sharpe index:

$$
\text { IS }=\frac{x_{p}}{\sigma_{x}}
$$

Where

$-x_{p}=$ return of asset $\mathrm{X}$;

- $\sigma_{x}=$ standard deviation of asset X.

It measures how much more return is given for each additional unit of risk.

\section{METHODOLOGY}

\subsection{General data}

The methodology involves the quantitative model analysis, exploring the financial and statistical data from the top 10 companies of BM \&, that is, companies with relatively large amounts of shares traded. This information was drawn from the 
INDEPENDENT JOURNAL OF MANAGEMENT \& PRODUCTION (IJM\&P)

http://www.ijmp.jor.br

v. 7, n. 1, January - March 2016

ISSN: 2236-269X

DOI: 10.14807/ijmp.v7i1.324

company's Thomson Reuters Eikon database, a leader in collection and distribution of information on the business market.

To carry out this work we considered the following periods as pre-crisis, crisis and post-crisis scenarios.

Table 1: Analyzed periods

\begin{tabular}{|c|c|}
\hline Period & Scenario \\
\hline January/2004 to June/2007 & Pre-crisis \\
\hline July/2007 to June/2009 & Crisis \\
\hline July/2009 to December/2012 & Post-crisis \\
\hline
\end{tabular}

Source: The author

The ten companies chosen for this study with their codes of their actions are listed in Table 2.

Table 2 - Analyzed companies

\begin{tabular}{|l|l|}
\hline Action Code & Company \\
\hline BBAS3.SA & Banco do Brasil \\
\hline BBDC4.SA & Banco Bradesco \\
\hline CCRO3.SA & Companhia de Concessões Rodoviárias \\
\hline CMIG4.SA & Companhia Energética de Minas Gerais, \\
\hline CSNA3.SA & Companhia Siderúrgica Nacional \\
\hline EMBR3.SA & Embraer \\
\hline GGB & Gerdau \\
\hline ITUB.K & Itaú Unibanco Holding \\
\hline PETR4.SA & Petrobrás \\
\hline VALE5.SA & Vale \\
\hline
\end{tabular}

Source: The author

\subsection{Analysis of covariance}

In order to identify the covariance behavior over time we structured semiannual covariance matrices between the companies' share returns. Each matrix has the covariances of returns of the companies within a semester over eight years (2004-2012). Furthermore, for matrices calculation we used the "COVARIAÇÃO.S" tool from the Microsoft Excel program. The result of this formula is the deviation average of products of each pair of points in two datasets, in this case, two sets return of two different companies. Therefore, the matrix is composed of covariances of all possible pairwise combinations of the ten aforementioned companies.

\subsubsection{Constructing hypothetical portfolio}

This section of the study primarily aims to quantify the influence of the covariances instability in a theoretical portfolio, by changing the assets weights over time. 
INDEPENDENT JOURNAL OF MANAGEMENT \& PRODUCTION (IJM\&P)

http://www.ijmp.jor.br

v. 7, n. 1, January - March 2016

ISSN: 2236-269X

DOI: 10.14807/ijmp.v7i1.324

First of all, we identified, for each of the three periods studied (pre-crisis, crisis and post-crisis) their returns, standard deviations, and covariance matrices. From these variables, we built six hypothetical portfolios. Three portfolios have restrictions such as the preference of a Sharpe index of a hypothetical investor equal to $15 \%$ in the three periods. The three other portfolios must maintain constant their weights, to quantify the Sharpe index variation.

In order to find the returns, standard deviations and covariance matrices, we used formulas showed in the theoretical foundation of this article. Whereas the construction of portfolios that meet a Sharpe index of $15 \%$ were made by the SOLVER tool from Microsoft Excel, under the following restrictions:

$$
\begin{aligned}
& -\quad W i \geq 0 \\
& -\quad \sum_{i=1}^{n} W i=1
\end{aligned}
$$

\section{ANALYSIS OF COVARIANCE}

The covariance matrices of the ten companies in the study are as follows.

\begin{tabular}{|c|c|c|c|c|c|c|c|c|c|c|}
\hline \multicolumn{11}{|c|}{ 10 SEMESTRE 2004} \\
\hline & BBAS3 & BBDC4 & CCRO3 & CMIG4 & CSNA3 & EMBR3 & GGB & ITUB.K & PETR4 & VALE5 \\
\hline BBAS3 & 0,13976 & 0,11425 & 0,00037 & 0,09142 & 0,15211 & 0,42324 & \begin{tabular}{|l|}
0,04299 \\
\end{tabular} & 0,09565 & 0,16317 & 0,20200 \\
\hline BBDC4 & 0,11425 & 0,12120 & 0,00091 & 0,09135 & 0,13951 & 0,40071 & 0,03578 & 0,09630 & 0,15204 & 0,21505 \\
\hline CCRO3 & 0,00037 & 0,00091 & 0,01229 & 0,01088 & 0,02338 & 0,01371 & 0,00772 & 0,00540 & 0,01979 & 0,02362 \\
\hline CMIG4 & 0,09142 & 0,09135 & 0,01088 & 0,10475 & 0,15355 & 0,36308 & \begin{tabular}{|l|l}
0,03320 \\
\end{tabular} & 0,08241 & 0,16759 & 0,15618 \\
\hline CSNA3 & 0,15211 & 0,13951 & 0,02338 & 0,15355 & 0,37979 & 0,58122 & 0,06413 & 0,12466 & 0,28518 & 0,24623 \\
\hline EMBR3 & 0,42324 & 0,40071 & 0,01371 & 0,36308 & 0,58122 & 1,71749 & \begin{tabular}{|l|}
0,14847 \\
\end{tabular} & 0,34412 & 0,61310 & 0,68965 \\
\hline GGBR4 & 0,04299 & 0,03578 & 0,00772 & 0,03320 & 0,06413 & 0,14847 & 0,03546 & 0,03228 & 0,05752 & 0,03202 \\
\hline ITSA4 & 0,09565 & 0,09630 & 0,00540 & 0,08241 & 0,12466 & 0,34412 & 0,03228 & 0,08837 & 0,14085 & 0,17904 \\
\hline PETR4 & 0,16317 & 0,15204 & 0,01979 & 0,16759 & 0,28518 & 0,61310 & 0,05752 & 0,14085 & 0,31301 & 0,27015 \\
\hline VALE5 & 0,20200 & 0,21505 & 0,02362 & 0,15618 & 0,24623 & 0,68965 & 0,03202 & 0,17904 & 0,27015 & 0,52373 \\
\hline
\end{tabular}

Table 3: Covariance matrix of $1^{\text {st }}$ semester of 2004

Source: The author

\begin{tabular}{|c|c|c|c|c|c|c|c|c|c|c|}
\hline \multicolumn{11}{|c|}{ 20 SEMESTRE 2004} \\
\hline & BBAS3 & BBDC4 & CCRO3 & CMIG4 & CSNA3 & EMBR3 & GGB & ITUB.K & PETR4 & VALE5 \\
\hline BBAS3 & 0,64856 & 0,50188 & 0,28974 & 0,32087 & 0,20998 & 0,37874 & 0,14621 & 0,41379 & 0,50461 & 0,80898 \\
\hline BBDC4 & 0,50188 & 0,42866 & 0,23101 & 0,25949 & 0,15590 & 0,27209 & 0,10620 & 0,33990 & 0,39141 & 0,62594 \\
\hline CCRO3 & 0,28974 & 0,23101 & 0,14453 & 0,13471 & 0,11106 & 0,11970 & 0,07363 & 0,19748 & 0,21035 & 0,37403 \\
\hline CMIG4 & 0,32087 & 0,25949 & 0,13471 & 0,20333 & 0,10143 & 0,26803 & 0,08462 & 0,21442 & 0,30566 & 0,45203 \\
\hline CSNA3 & 0,20998 & 0,15590 & 0,11106 & 0,10143 & 0,14227 & 0,04589 & 0,09791 & 0,14553 & 0,15100 & 0,31666 \\
\hline EMBR3 & 0,37874 & 0,27209 & 0,11970 & 0,26803 & 0,04589 & 0,83688 & 0,10197 & 0,23487 & 0,52520 & 0,58181 \\
\hline GGBR4 & 0,14621 & 0,10620 & 0,07363 & 0,08462 & 0,09791 & 0,10197 & 0,10318 & 0,11659 & 0,15830 & 0,27617 \\
\hline ITSA4 & 0,41379 & 0,33990 & 0,19748 & 0,21442 & 0,14553 & 0,23487 & 0,11659 & 0,29874 & 0,35698 & 0,58470 \\
\hline $\begin{array}{l}\text { PETR4 } \\
\end{array}$ & 0,50461 & 0,39141 & 0,21035 & 0,30566 & 0,15100 & 0,52520 & 0,15830 & 0,35698 & 0,57686 & 0,80603 \\
\hline VALE5 & 0,80898 & 0,62594 & 0,37403 & 0,45203 & 0,31666 & 0,58181 & 0,27617 & 0,58470 & 0,80603 & 1,31350 \\
\hline
\end{tabular}

Table 4: Covariance matrix of $2^{\text {nd }}$ semester 2004

Source: The author 
INDEPENDENT JOURNAL OF MANAGEMENT \& PRODUCTION (IJM\&P)

http://www.ijmp.jor.br

v. 7, n. 1, January - March 2016

ISSN: 2236-269X

DOI: 10.14807/ijmp.v7i1.324

Table 5: Covariance matrix of $1^{\text {st }}$ semester of 2005

\begin{tabular}{|c|c|c|c|c|c|c|c|c|c|c|}
\hline \multicolumn{11}{|c|}{ 10 SEMESTRE 2005} \\
\hline & BBAS3 & BBDC4 & CCRO3 & CMIG4 & CSNA3 & EMBR3 & GGB & ITUB.K & $\begin{array}{l}\text { PETR4 } \\
\end{array}$ & VALE5 \\
\hline BBAS3 & 0,14786 & 0,00854 & 0,00749 & 0,02390 & 0,08254 & 0,14902 & 0,03161 & 0,00307 & 0,07713 & 0,11558 \\
\hline BBDC4 & 0,00854 & 0,57346 & 0,00155 & 0,19637 & 0,08807 & 0,14799 & 0,01299 & 0,27275 & 0,32895 & 0,10350 \\
\hline CCRO3 & 0,00749 & 0,00155 & 0,01340 & 0,00338 & 0,00165 & 0,04050 & 0,01156 & 0,00508 & 0,02219 & 0,02741 \\
\hline CMIG4 & 0,02390 & 0,19637 & 0,00338 & 0,15862 & 0,17742 & 0,09033 & 0,08779 & 0,11227 & 0,06689 & 0,20827 \\
\hline CSNA3 & 0,08254 & 0,08807 & 0,00165 & 0,17742 & 1,07562 & 0,62375 & 0,32641 & 0,08363 & 0,23824 & 1,10049 \\
\hline EMBR3 & 0,14902 & $\begin{array}{l}0,14799 \\
\end{array}$ & 0,04050 & 0,09033 & 0,62375 & 0,71186 & \begin{tabular}{|l|}
0,23891 \\
\end{tabular} & 0,03237 & 0,33896 & 0,77870 \\
\hline GGBR4 & 0,03161 & 0,01299 & 0,01156 & 0,08779 & 0,32641 & 0,23891 & 0,14799 & 0,01627 & 0,07896 & 0,35271 \\
\hline ITSA4 & 0,00307 & 0,27275 & 0,00508 & 0,11227 & 0,08363 & 0,03237 & 0,01627 & 0,17005 & 0,15474 & 0,06616 \\
\hline PETR4 & 0,07713 & 0,32895 & 0,02219 & 0,06689 & 0,23824 & 0,33896 & 0,07896 & 0,15474 & 0,38873 & 0,38035 \\
\hline VALE5 & 0,11558 & 0,10350 & 0,02741 & 0,20827 & 1,10049 & 0,77870 & 0,35271 & 0,06616 & 0,38035 & 1,34771 \\
\hline
\end{tabular}

Source: The author

Table 6: Covariance matrix of $2^{\text {nd }}$ semester of 2005

\begin{tabular}{|c|c|c|c|c|c|c|c|c|c|c|}
\hline \multicolumn{11}{|c|}{ 20 SEMESTRE 2005} \\
\hline & BBAS3 & BBDC4 & CCRO3 & CMIG4 & CSNA3 & EMBR3 & GGB & ITUB.K & PETR4 & VALE5 \\
\hline BBAS3 & 0,99678 & 1,72126 & 0,23806 & 0,29896 & 0,21663 & 0,60271 & 0,62057 & 0,77516 & 1,07993 & 1,41330 \\
\hline BBDC4 & 1,72126 & 4,07016 & 0,59276 & 0,64877 & 0,36734 & 1,29271 & 1,25005 & 1,63812 & 2,17470 & 2,63051 \\
\hline CCRO3 & 0,23806 & 0,59276 & 0,10062 & 0,10011 & 0,05041 & 0,21639 & 0,19086 & 0,24514 & 0,32078 & 0,36927 \\
\hline CMIG4 & 0,29896 & 0,64877 & 0,10011 & 0,16006 & 0,13308 & 0,27138 & 0,23580 & 0,26584 & 0,47956 & 0,48064 \\
\hline CSNA3 & 0,21663 & 0,36734 & 0,05041 & 0,13308 & 0,18244 & 0,21190 & 0,18040 & 0,15971 & 0,42425 & 0,36817 \\
\hline EMBR3 & 0,60271 & 1,29271 & 0,21639 & 0,27138 & 0,21190 & 0,77816 & 0,50736 & 0,53947 & 0,91594 & 0,85563 \\
\hline GGBR4 & 0,62057 & 1,25005 & 0,19086 & 0,23580 & 0,18040 & 0,50736 & 0,48131 & 0,57144 & 0,81582 & 0,93038 \\
\hline ITSA4 & 0,77516 & 1,63812 & 0,24514 & 0,26584 & 0,15971 & 0,53947 & 0,57144 & 0,77098 & 0,93297 & 1,17970 \\
\hline PETR4 & 1,07993 & 2,17470 & 0,32078 & 0,47956 & 0,42425 & 0,91594 & 0,81582 & 0,93297 & 1,71862 & 1,70859 \\
\hline VALE5 & 1,41330 & 2,63051 & 0,36927 & 0,48064 & 0,36817 & 0,85563 & 0,93038 & 1,17970 & 1,70859 & 2,26387 \\
\hline
\end{tabular}

Source: The author

Table 7: Covariance matrix of $1^{\text {st }}$ semester of 2006

\begin{tabular}{|c|c|c|c|c|c|c|c|c|c|c|}
\hline \multicolumn{11}{|c|}{ 19 SEMESTRE 2006} \\
\hline & BBAS3 & BBDC4 & CCRO3 & CMIG4 & CSNA3 & EMBR3 & GGB & ITUB.K & PETR4 & VALE5 \\
\hline BBAS3 & 0,91431 & 0,74643 & 0,04024 & 0,13731 & 0,33277 & 0,13474 & 0,47867 & 0,53313 & 0,58171 & 0,37023 \\
\hline BBDC4 & 0,74643 & 2,36171 & 0,29970 & 0,65302 & 0,16667 & 0,74102 & 0,38252 & 0,88887 & 0,90018 & 0,80541 \\
\hline CCRO3 & 0,04024 & 0,29970 & 0,09673 & 0,11935 & 0,08782 & 0,18634 & 0,07176 & 0,01374 & 0,07494 & 0,15332 \\
\hline CMIG4 & 0,13731 & 0,65302 & 0,11935 & 0,28975 & 0,06071 & 0,25450 & 0,07235 & 0,10324 & 0,24364 & 0,34343 \\
\hline CSNA3 & 0,33277 & 0,16667 & 0,08782 & 0,06071 & 0,55456 & 0,14215 & 0,51322 & 0,36640 & 0,43826 & 0,23457 \\
\hline EMBR3 & 0,13474 & 0,74102 & 0,18634 & 0,25450 & 0,14215 & 0,82973 & 0,08886 & 0,11435 & 0,21765 & 0,22611 \\
\hline GGBR4 & 0,47867 & 0,38252 & 0,07176 & 0,07235 & 0,51322 & 0,08886 & 0,84355 & 0,74880 & 0,38300 & 0,12264 \\
\hline ITSA4 & 0,53313 & 0,88887 & 0,01374 & 0,10324 & 0,36640 & 0,11435 & 0,74880 & 0,99294 & 0,41380 & 0,16951 \\
\hline PETR4 & 0,58171 & 0,90018 & 0,07494 & 0,24364 & 0,43826 & 0,21765 & 0,38300 & 0,41380 & 0,88353 & 0,78227 \\
\hline VALE5 & 0,37023 & 0,80541 & 0,15332 & 0,34343 & 0,23457 & 0,22611 & 0,12264 & 0,16951 & 0,78227 & 1,04537 \\
\hline
\end{tabular}

Source: The author

Table 8: Covariance matrix of $2^{\text {nd }}$ semester of 2006

\begin{tabular}{|c|c|c|c|c|c|c|c|c|c|c|}
\hline \multicolumn{11}{|c|}{ 20 SEMESTRE 2006} \\
\hline & BBAS3 & BBDC4 & CCRO3 & CMIG4 & CSNA3 & EMBR3 & GGB & ITUB.K & PETR4 & VALE5 \\
\hline BBAS3 & 1,37130 & 0,74643 & 0,69311 & 0,36081 & 0,06893 & 0,91140 & 0,23478 & 0,66043 & 0,83426 & 1,94974 \\
\hline BBDC4 & 1,17289 & 1,31008 & 0,67595 & 0,29520 & 0,08279 & 1,22776 & 0,14248 & 0,72975 & 0,53635 & 1,69341 \\
\hline CCRO3 & 0,69311 & 0,67595 & 0,41101 & 0,16231 & 0,08650 & 0,66275 & 0,10133 & 0,40131 & 0,31675 & 0,93522 \\
\hline CMIG4 & 0,36081 & 0,29520 & 0,16231 & 0,13126 & 0,03092 & 0,15555 & 0,06943 & 0,15723 & 0,29658 & 0,60819 \\
\hline CSNA3 & 0,06893 & 0,08279 & 0,08650 & 0,03092 & 0,12142 & 0,23705 & 0,00244 & 0,06338 & 0,11092 & 0,06248 \\
\hline EMBR3 & 0,91140 & 1,22776 & 0,66275 & 0,15555 & 0,23705 & 2,00436 & 0,06193 & 0,73338 & 0,16400 & 1,11316 \\
\hline GGBR4 & 0,23478 & 0,14248 & 0,10133 & 0,06943 & 0,00244 & 0,06193 & 0,17395 & 0,16058 & 0,30398 & 0,36304 \\
\hline ITSA4 & 0,66043 & 0,72975 & 0,40131 & 0,15723 & 0,06338 & 0,73338 & 0,16058 & 0,60894 & 0,30288 & 0,99574 \\
\hline PETR4 & 0,83426 & 0,53635 & 0,31675 & 0,29658 & 0,11092 & 0,16400 & 0,30398 & 0,30288 & 1,04439 & 1,42515 \\
\hline VALE5 & 1,94974 & 1,69341 & 0,93522 & 0,60819 & 0,06248 & 1,11316 & 0,36304 & 0,99574 & 1,42515 & 3,21538 \\
\hline
\end{tabular}

Source: The author

Table 9: Covariance matrix of $1^{\text {st }}$ semester of 2007

\begin{tabular}{|c|c|c|c|c|c|c|c|c|c|c|}
\hline \multicolumn{11}{|c|}{ 10 SEMESTRE 2007} \\
\hline & BBAS3 & BBDC4 & CCRO3 & CMIG4 & CSNA3 & EMBR3 & GGB & ITUB.K & PETR4 & VALE5 \\
\hline BBAS3 & 1,95447 & 1,55059 & 0,59248 & 0,73458 & 1,79245 & 0,36449 & 1,52807 & 1,44009 & 0,94343 & 2,95263 \\
\hline BBDC4 & 1,55059 & 1,71527 & 0,50276 & 0,55347 & 1,24199 & 0,03416 & 1,04596 & 1,13766 & 0,91346 & 2,02145 \\
\hline CCRO3 & 0,59248 & 0,50276 & 0,23526 & 0,24036 & 0,59534 & 0,14763 & 0,50210 & 0,41877 & 0,34857 & 1,02566 \\
\hline CMIG4 & 0,73458 & 0,55347 & 0,24036 & 0,32861 & 0,68090 & 0,18940 & 0,63587 & 0,55590 & 0,44370 & 1,16171 \\
\hline CSNA3 & 1,79245 & 1,24199 & 0,59534 & 0,68090 & 2,41257 & 0,80712 & 1,59366 & 1,21309 & 0,73249 & 3,91059 \\
\hline EMBR3 & 0,36449 & 0,03416 & 0,14763 & 0,18940 & 0,80712 & 0,68389 & 0,55657 & 0,26696 & 0,02220 & 1,50008 \\
\hline GGBR4 & 1,52807 & 1,04596 & 0,50210 & 0,63587 & 1,59366 & 0,55657 & 1,49018 & 1,23548 & 0,67336 & 2,64549 \\
\hline ITSA4 & 1,44009 & 1,13766 & 0,41877 & 0,55590 & 1,21309 & 0,26696 & 1,23548 & 1,29444 & 0,69618 & 1,95588 \\
\hline $\begin{array}{l}\text { PETR4 } \\
\end{array}$ & 0,94343 & 0,91346 & 0,34857 & 0,44370 & 0,73249 & 0,02220 & 0,67336 & 0,69618 & 1,04347 & 1,22648 \\
\hline VALE5 & 2,95263 & 2,02145 & 1,02566 & 1,16171 & 3,91059 & 1,50008 & 2,64549 & 1,95588 & 1,22648 & 6,97819 \\
\hline
\end{tabular}

Source: The author 
INDEPENDENT JOURNAL OF MANAGEMENT \& PRODUCTION (IJM\&P)

http://www.ijmp.jor.br

v. 7, n. 1, January - March 2016

ISSN: 2236-269X

DOI: 10.14807/ijmp.v7i1.324

Table 10: Covariance matrix of $2^{\text {nd }}$ semester of 2007

\begin{tabular}{|c|c|c|c|c|c|c|c|c|c|c|}
\hline \multicolumn{11}{|c|}{ 20 SEMESTRE 2007} \\
\hline & BBAS3 & BBDC4 & CCRO3 & CMIG4 & CSNA3 & EMBR3 & GGB & ITUB.K & PETR4 & VALE5 \\
\hline BBAS3 & 1,10353 & 1,30060 & 0,03283 & 0,12836 & 1,16506 & 0,16569 & 0,32025 & 0,40727 & 2,23637 & 2,69811 \\
\hline BBDC4 & 1,30060 & 2,91733 & 0,33776 & 0,07581 & 2,98669 & 0,15428 & 1,24517 & 1,63211 & 6,63272 & 7,34219 \\
\hline CCRO3 & 0,03283 & 0,33776 & 0,28072 & 0,18061 & 0,60070 & 0,20966 & 0,40561 & 0,59232 & 1,81161 & 1,11298 \\
\hline CMIG4 & 0,12836 & 0,07581 & 0,18061 & 0,22560 & 0,35636 & 0,23671 & 0,12199 & 0,25331 & 0,85223 & 0,40863 \\
\hline CSNA3 & 1,16506 & 2,98669 & 0,60070 & 0,35636 & 3,90496 & 0,50438 & 1,12836 & 1,80241 & 8,40760 & 8,86823 \\
\hline EMBR3 & 0,16569 & 0,15428 & 0,20966 & 0,23671 & 0,50438 & 0,82725 & 0,23565 & 0,24525 & 1,36299 & 1,31884 \\
\hline GGBR4 & 0,32025 & 1,24517 & 0,40561 & 0,12199 & 1,12836 & 0,23565 & 1,75059 & 2,02726 & 4,09370 & 2,75780 \\
\hline ITSA4 & 0,40727 & 1,63211 & 0,59232 & 0,25331 & 1,80241 & 0,24525 & 2,02726 & 2,67194 & 5,57878 & 4,13921 \\
\hline PETR4 & 2,23637 & 6,63272 & 1,81161 & 0,85223 & 8,40760 & 1,36299 & 4,09370 & 5,57878 & 23,11169 & 19,53912 \\
\hline VALE5 & 2,69811 & 7,34219 & 1,11298 & 0,40863 & 8,86823 & 1,31884 & 2,75780 & 4,13921 & 19,53912 & 24,52083 \\
\hline
\end{tabular}

Source: The author

Table 11: Covariance matrix of $1^{\text {st }}$ semester of 2008

\begin{tabular}{|c|c|c|c|c|c|c|c|c|c|c|}
\hline \multicolumn{11}{|c|}{ 10 SEMESTRE 2008} \\
\hline & BBAS3 & BBDC4 & CCRO3 & CMIG4 & CSNA3 & EMBR3 & GGB & ITUB.K & PETR4 & VALE5 \\
\hline BBAS3 & 2,12778 & 0,82518 & 0,38739 & 0,57793 & 0,86635 & 0,28203 & 0,97898 & 0,76947 & 2,72939 & 0,72102 \\
\hline BBDC4 & 0,82518 & 2,34019 & 0,52708 & 1,04348 & 4,20005 & 0,89839 & 2,08556 & 0,32205 & 3,58118 & 3,63548 \\
\hline CCRO3 & 0,38739 & 0,52708 & 0,33101 & 0,46092 & 1,57699 & 0,84673 & 1,61526 & 0,38926 & 1,47475 & 0,80937 \\
\hline CMIG4 & 0,57793 & 1,04348 & 0,46092 & 0,88828 & 2,63096 & 1,53501 & 2,85297 & 0,81148 & 2,46916 & 1,48113 \\
\hline CSNA3 & 0,86635 & 4,20005 & 1,57699 & 2,63096 & 13,42529 & 4,47486 & 7,75553 & 0,85962 & 9,58663 & 8,34521 \\
\hline EMBR3 & 0,28203 & 0,89839 & 0,84673 & 1,53501 & 4,47486 & 4,26714 & 6,86607 & 1,89321 & 3,33189 & 0,72964 \\
\hline GGBR4 & 0,97898 & 2,08556 & 1,61526 & 2,85297 & 7,75553 & 6,86607 & 12,76269 & 4,10880 & 6,71522 & 2,43176 \\
\hline ITSA4 & 0,76947 & 0,32205 & 0,38926 & 0,81148 & 0,85962 & 1,89321 & 4,10880 & 2,36199 & 1,80052 & 0,00355 \\
\hline PETR4 & 2,72939 & 3,58118 & 1,47475 & 2,46916 & 9,58663 & 3,33189 & 6,71522 & 1,80052 & 11,00839 & 6,69479 \\
\hline VALE5 & 0,72102 & 3,63548 & 0,80937 & 1,48113 & 8,34521 & 0,72964 & 2,43176 & 0,00355 & 6,69479 & 8,21875 \\
\hline
\end{tabular}

Source: The author

Table 12: Covariance matrix of $2^{\text {nd }}$ semester of 2008

\begin{tabular}{|c|c|c|c|c|c|c|c|c|c|c|}
\hline \multicolumn{11}{|c|}{ 20 SEMESTRE 2008} \\
\hline & BBAS3 & BBDC4 & CCRO3 & CMIG4 & CSNA3 & EMBR3 & GGB & ITUB.K & PETR4 & VALE5 \\
\hline BBAS3 & 7,72622 & 6,27814 & 1,88239 & 0,92553 & 13,64128 & 3,37084 & 18,27491 & 9,07395 & 15,15383 & 14,07742 \\
\hline BBDC4 & 6,27814 & 5,47312 & 1,56032 & 0,85640 & 11,15312 & 2,54475 & 14,60481 & 7,02226 & 12,34112 & 11,51012 \\
\hline CCRO3 & 1,88239 & 1,56032 & 0,57868 & 0,25522 & 3,48150 & 0,71373 & \begin{tabular}{|l|}
4,35487 \\
\end{tabular} & 2,02395 & 3,54119 & 3,51304 \\
\hline CMIG4 & 0,92553 & 0,85640 & 0,25522 & 0,41635 & 1,73620 & 0,04110 & 2,18008 & 0,84860 & 1,92916 & 1,88641 \\
\hline CSNA3 & 13,64128 & 11,15312 & 3,48150 & 1,73620 & 26,20954 & 5,27812 & 34,50097 & 16,85438 & 28,25051 & 26,70725 \\
\hline EMBR3 & 3,37084 & 2,54475 & 0,71373 & 0,04110 & 5,27812 & 2,68585 & 7,62911 & \begin{tabular}{|l|}
4,28697 \\
\end{tabular} & 5,87474 & 5,26867 \\
\hline GGBR4 & 18,27491 & 14,60481 & 4,35487 & 2,18008 & 34,50097 & 7,62911 & 50,75190 & 25,77196 & 38,51761 & 34,92815 \\
\hline ITSA4 & 9,07395 & 7,02226 & 2,02395 & 0,84860 & 16,85438 & 4,28697 & 25,77196 & 13,97273 & 19,20479 & 17,04780 \\
\hline PETR4 & 15,15383 & 12,34112 & 3,54119 & 1,92916 & 28,25051 & 5,87474 & 38,51761 & 19,20479 & 34,00734 & 29,82375 \\
\hline VALE5 & 14,07742 & 11,51012 & 3,51304 & 1,88641 & 26,70725 & 5,26867 & 34,92815 & 17,04780 & 29,82375 & 28,23413 \\
\hline
\end{tabular}

Source: The author

Table 13: Covariance matrix of $1^{\text {st }}$ semester of 2009

\begin{tabular}{|c|c|c|c|c|c|c|c|c|c|c|}
\hline \multicolumn{11}{|c|}{ 10 SEMESTRE 2009} \\
\hline & BBAS3 & BBDC4 & CCRO3 & CMIG4 & CSNA3 & EMBR3 & GGB & ITUB.K & PETR4 & VALE5 \\
\hline BBAS3 & 4,97446 & 4,72829 & 1,23288 & 0,70893 & 4,64459 & 0,52488 & 0,68810 & 1,87534 & 6,15330 & 3,69955 \\
\hline BBDC4 & 4,72829 & 4,80855 & 1,26065 & 0,65862 & 4,63667 & 0,78386 & 0,69970 & 1,90940 & 5,88143 & 3,88590 \\
\hline CCRO3 & 1,23288 & 1,26065 & 0,41390 & 0,15382 & 1,27835 & 0,24503 & 0,40182 & 0,75307 & 1,41097 & 1,04435 \\
\hline CMIG4 & 0,70893 & 0,65862 & 0,15382 & 0,14061 & 0,62646 & 0,05766 & 0,06232 & 0,21036 & 0,87905 & 0,46476 \\
\hline CSNA3 & 4,64459 & 4,63667 & 1,27835 & 0,62646 & 5,00449 & 0,88044 & 0,97980 & 2,23040 & 5,84075 & 4,22374 \\
\hline EMBR3 & 0,52488 & 0,78386 & 0,24503 & 0,05766 & 0,88044 & 1,05649 & 0,16319 & 0,42860 & 0,23845 & 0,80982 \\
\hline GGBR4 & 0,68810 & 0,69970 & 0,40182 & 0,06232 & 0,97980 & 0,16319 & 1,38387 & 1,34977 & 0,79628 & 0,80861 \\
\hline ITSA4 & 1,87534 & 1,90940 & 0,75307 & 0,21036 & 2,23040 & 0,42860 & 1,34977 & 2,06206 & 1,89831 & 1,68284 \\
\hline PETR4 & 6,15330 & 5,88143 & 1,41097 & 0,87905 & 5,84075 & 0,23845 & 0,79628 & 1,89831 & 8,71967 & 5,09480 \\
\hline VALE5 & 3,69955 & 3,88590 & 1,04435 & 0,46476 & 4,22374 & 0,80982 & 0,80861 & 1,68284 & 5,09480 & 4,32041 \\
\hline
\end{tabular}

Source: The author

Table 14: Covariance matrix of $2^{\text {nd }}$ semester of 2009

\begin{tabular}{|c|c|c|c|c|c|c|c|c|c|c|}
\hline \multicolumn{11}{|c|}{ 20 SEMESTRE 2009} \\
\hline & BBAS3 & BBDC4 & CCRO3 & CMIG4 & CSNA3 & EMBR3 & GGB & ITUB.K & PETR4 & VALE5 \\
\hline BBAS3 & 6,48752 & 5,23065 & 1,34217 & 0,96436 & 5,47145 & 0,70238 & \begin{tabular}{|l|}
4,18699 \\
\end{tabular} & 5,05748 & 5,54585 & 9,15269 \\
\hline BBDC4 & 5,23065 & 4,77715 & 1,39568 & 1,02959 & 4,67192 & 0,29974 & 4,01282 & 4,67541 & 4,97745 & 8,29724 \\
\hline CCRO3 & 1,34217 & 1,39568 & 0,58866 & 0,40730 & 1,28176 & 0,13812 & 1,61047 & 1,73529 & 1,60896 & 2,69423 \\
\hline CMIG4 & 0,96436 & 1,02959 & 0,40730 & 0,37875 & 0,93061 & 0,01728 & 1,12373 & 1,16467 & 1,16014 & 1,87067 \\
\hline CSNA3 & 5,47145 & 4,67192 & 1,28176 & 0,93061 & 5,27897 & 0,45883 & 3,71879 & 4,49624 & 5,17922 & 8,66868 \\
\hline EMBR3 & 0,70238 & 0,29974 & 0,13812 & 0,01728 & 0,45883 & 0,62430 & 0,29452 & 0,10478 & 0,07070 & 0,18411 \\
\hline GGBR4 & 4,18699 & 4,01282 & 1,61047 & 1,12373 & 3,71879 & 0,29452 & 5,31814 & 5,56190 & 4,86126 & 7,95266 \\
\hline ITSA4 & 5,05748 & 4,67541 & 1,73529 & 1,16467 & 4,49624 & 0,10478 & 5,56190 & 6,24557 & 5,37819 & 9,15391 \\
\hline PETR4 & 5,54585 & 4,97745 & 1,60896 & 1,16014 & 5,17922 & 0,07070 & 4,86126 & 5,37819 & 5,90644 & 9,45604 \\
\hline VALE5 & 9,15269 & 8,29724 & 2,69423 & 1,87067 & 8,66868 & 0,18411 & 7,95266 & 9,15391 & 9,45604 & 15,97958 \\
\hline
\end{tabular}

Source: The author 
INDEPENDENT JOURNAL OF MANAGEMENT \& PRODUCTION (IJM\&P)

http://www.ijmp.jor.br

v. 7, n. 1, January - March 2016

ISSN: 2236-269X

DOI: 10.14807/ijmp.v7i1.324

Table 15: Covariance matrix of $1^{\text {st }}$ semester of 2010

\begin{tabular}{|c|c|c|c|c|c|c|c|c|c|c|}
\hline \multicolumn{11}{|c|}{ 10 SEMESTRE 2010} \\
\hline & BBAS3 & BBDC4 & CCRO3 & CMIG4 & CSNA3 & EMBR3 & $\overline{G G B}$ & ITUB.K & PETR4 & VALE5 \\
\hline BBAS3 & 1,08944 & 0,73256 & 0,19359 & 0,00155 & 1,73424 & 0,17111 & 0,46600 & 0,21039 & 2,12857 & 2,34394 \\
\hline BBDC4 & 0,73256 & 0,85974 & 0,25670 & 0,04373 & 1,03349 & 0,07978 & \begin{tabular}{|c|}
0,14926 \\
\end{tabular} & 0,11200 & 2,01804 & 1,77293 \\
\hline CCRO3 & 0,19359 & 0,25670 & 0,14372 & 0,02394 & 0,27534 & 0,02265 & 0,05263 & 0,06537 & 0,73855 & 0,55989 \\
\hline CMIG4 & 0,00155 & 0,04373 & 0,02394 & 0,07343 & 0,03003 & 0,00449 & 0,02209 & 0,04939 & 0,00338 & 0,03164 \\
\hline CSNA3 & 1,73424 & 1,03349 & 0,27534 & 0,03003 & 5,09103 & 0,57168 & 1,97714 & 1,37557 & 3,17656 & 5,58419 \\
\hline EMBR3 & 0,17111 & 0,07978 & 0,02265 & 0,00449 & 0,57168 & 0,09328 & 0,21785 & 0,15104 & 0,25836 & 0,58616 \\
\hline GGBR4 & 0,46600 & 0,14926 & 0,05263 & 0,02209 & 1,97714 & 0,21785 & 1,80335 & 1,41815 & 0,87970 & 1,68629 \\
\hline ITSA4 & 0,21039 & 0,11200 & 0,06537 & 0,04939 & 1,37557 & 0,15104 & 1,41815 & 1,31919 & 0,18726 & 0,92683 \\
\hline PETR4 & 2,12857 & 2,01804 & 0,73855 & 0,00338 & 3,17656 & 0,25836 & 0,87970 & 0,18726 & 6,97530 & 4,96873 \\
\hline VALE5 & 2,34394 & 1,77293 & 0,55989 & 0,03164 & 5,58419 & 0,58616 & 1,68629 & 0,92683 & 4,96873 & 7,34228 \\
\hline
\end{tabular}

Source: The author

Table 16: Covariance matrix of $2^{\text {nd }}$ semester of 2010

\begin{tabular}{|c|c|c|c|c|c|c|c|c|c|c|}
\hline \multicolumn{11}{|c|}{ 20 SEMESTRE 2010} \\
\hline & BBAS3 & BBDC4 & CCRO3 & CMIG4 & CSNA3 & EMBR3 & GGB & ITUB.K & PETR4 & VALE5 \\
\hline BBAS3 & 3,40240 & 3,42213 & 1,39321 & 0,70688 & 0,32427 & 1,17502 & 0,00087 & 2,49599 & 0,80192 & 5,41290 \\
\hline BBDC4 & 3,42213 & 3,66904 & 1,43975 & 0,74256 & 0,46996 & 1,31222 & 0,21248 & 2,59831 & 0,68891 & 5,59098 \\
\hline CCRO3 & 1,39321 & 1,43975 & 0,73127 & 0,36650 & 0,01583 & 0,58101 & 0,01583 & 1,46768 & 0,42569 & 2,59975 \\
\hline CMIG4 & 0,70688 & 0,74256 & 0,36650 & 0,25356 & 0,04109 & 0,29455 & 0,02508 & 0,81171 & 0,26463 & 1,34494 \\
\hline CSNA3 & 0,32427 & 0,46996 & 0,01583 & 0,04109 & 0,67079 & 0,14690 & 0,21385 & 0,53972 & 0,43702 & 0,15393 \\
\hline EMBR3 & 1,17502 & 1,31222 & 0,58101 & 0,29455 & 0,14690 & 0,71667 & 0,09043 & 1,51817 & 0,21939 & 2,51005 \\
\hline GGBR4 & 0,00087 & 0,21248 & 0,01583 & 0,02508 & 0,21385 & 0,09043 & 0,51314 & 0,09650 & 0,17555 & 0,32831 \\
\hline ITSA4 & 2,49599 & 2,59831 & 1,46768 & 0,81171 & 0,53972 & 1,51817 & 0,09650 & 4,96337 & 1,15739 & 6,28677 \\
\hline PETR4 & 0,80192 & 0,68891 & 0,42569 & 0,26463 & 0,43702 & 0,21939 & 0,17555 & 1,15739 & 0,97604 & 1,54011 \\
\hline VALE5 & 5,41290 & 5,59098 & 2,59975 & 1,34494 & 0,15393 & 2,51005 & 0,32831 & 6,28677 & 1,54011 & 11,47598 \\
\hline
\end{tabular}

Source: The author

Table 17: Covariance matrix of $1^{\text {st }}$ semester of 2011

\begin{tabular}{|c|c|c|c|c|c|c|c|c|c|c|}
\hline \multicolumn{11}{|c|}{ 10 SEMESTRE 2011} \\
\hline & BBAS3 & BBDC4 & CCRO3 & CMIG4 & CSNA3 & EMBR3 & GGB & ITUB.K & PETR4 & VALE5 \\
\hline BBAS3 & 0,79822 & 0,59042 & 0,04161 & 0,36680 & 1,46740 & 0,11869 & 0,25946 & 0,16578 & 0,78162 & 1,67941 \\
\hline BBDC4 & 0,59042 & 0,65341 & 0,00337 & 0,05542 & 0,79986 & 0,01671 & 0,15687 & 0,01190 & 0,59703 & 0,88418 \\
\hline CCRO3 & 0,04161 & 0,00337 & 0,08936 & 0,17132 & 0,19877 & 0,04529 & 0,04623 & 0,04959 & 0,15646 & 0,32945 \\
\hline CMIG4 & 0,36680 & 0,05542 & 0,17132 & 0,70559 & 1,37979 & 0,28497 & 0,45119 & 0,17199 & 0,85557 & 1,58893 \\
\hline CSNA3 & 1,46740 & 0,79986 & 0,19877 & 1,37979 & 4,59986 & 0,75149 & 1,26877 & 0,06779 & 2,76336 & 4,44762 \\
\hline EMBR3 & 0,11869 & 0,01671 & 0,04529 & 0,28497 & 0,75149 & 0,38654 & 0,43554 & 0,09248 & 0,63768 & 0,58966 \\
\hline GGBR4 & 0,25946 & 0,15687 & 0,04623 & 0,45119 & 1,26877 & 0,43554 & 1,00068 & 0,11815 & 1,21954 & 0,95741 \\
\hline ITSA4 & 0,16578 & 0,01190 & 0,04959 & 0,17199 & 0,06779 & 0,09248 & 0,11815 & 0,76527 & 0,31299 & 0,49460 \\
\hline PETR4 & 0,78162 & 0,59703 & 0,15646 & 0,85557 & 2,76336 & 0,63768 & 1,21954 & 0,31299 & 2,52832 & 2,47082 \\
\hline VALE5 & 1,67941 & 0,88418 & 0,32945 & 1,58893 & 4,44762 & 0,58966 & 0,95741 & 0,49460 & 2,47082 & 5,55003 \\
\hline
\end{tabular}

Source: The author

Table 18: Covariance matrix of $2^{\text {nd }}$ semester of 2011

\begin{tabular}{|c|c|c|c|c|c|c|c|c|c|c|}
\hline \multicolumn{11}{|c|}{ 20 SEMESTRE 2011} \\
\hline & BBAS3 & BBDC4 & CCRO3 & CMIG4 & CSNA3 3 & EMBR3 & GGB & ITUB.K & PETR4 & VALE5 \\
\hline BBAS3 & 0,94184 & 0,32735 & 0,01359 & 0,06451 & 0,79585 & 0,01943 & 0,49539 & 0,83722 & 0,43950 & 1,24707 \\
\hline BBDC4 & 0,32735 & 1,84759 & 0,25530 & 0,73496 & 0,68553 & 0,77422 & 0,29217 & 0,91346 & 1,23619 & 1,11320 \\
\hline CCRO3 & 0,01359 & 0,25530 & 0,19628 & 0,04502 & 0,07708 & 0,19559 & 0,26257 & 0,61149 & 0,09010 & 0,15141 \\
\hline CMIG4 & 0,06451 & 0,73496 & 0,04502 & 0,66208 & 0,30233 & 0,13216 & 0,09352 & 0,13238 & 0,83090 & 0,39456 \\
\hline CSNA3 & 0,79585 & 0,68553 & 0,07708 & 0,30233 & 1,22668 & 0,31416 & 0,49297 & 0,68831 & 0,98532 & 2,03871 \\
\hline EMBR3 & 0,01943 & 0,77422 & 0,19559 & 0,13216 & 0,31416 & 0,78836 & 0,47663 & 1,19702 & 0,26121 & 0,74284 \\
\hline GGBR4 & 0,49539 & 0,29217 & 0,26257 & 0,09352 & 0,49297 & 0,47663 & 1,30919 & 2,48687 & 0,34631 & 0,61319 \\
\hline ITSA4 & 0,83722 & 0,91346 & 0,61149 & 0,13238 & 0,68831 & 1,19702 & 2,48687 & 5,25696 & 0,52274 & 0,69582 \\
\hline $\begin{array}{l}\text { PETR4 } \\
\end{array}$ & 0,43950 & 1,23619 & 0,09010 & 0,83090 & 0,98532 & 0,26121 & 0,34631 & 0,52274 & 1,88634 & 1,84458 \\
\hline VALE5 & 1,24707 & 1,11320 & 0,15141 & 0,39456 & 2,03871 & 0,74284 & 0,61319 & 0,69582 & 1,84458 & 4,26115 \\
\hline
\end{tabular}

Source: The author

Table 19: Covariance matrix of $1^{\text {st }}$ semester of 2012

\begin{tabular}{|c|c|c|c|c|c|c|c|c|c|c|}
\hline \multicolumn{11}{|c|}{ 10 SEMESTRE 2012} \\
\hline & BBAS3 & BBDC4 & CCRO3 & CMIG4 & CSNA3 & EMBR3 & GGB & ITUB.K & PETR4 & VALE5 \\
\hline BBAS3 & 6,67060 & 2,04988 & 1,84880 & 3,13166 & 4,06216 & 1,14158 & 0,66529 & 2,02945 & 5,13989 & 3,17027 \\
\hline BBDC4 & 2,04988 & 1,18492 & 0,71320 & 1,18599 & 1,23358 & 0,66608 & 0,29070 & 0,18684 & 1,77561 & 1,08012 \\
\hline CCRO3 & 1,84880 & 0,71320 & 1,87426 & 2,91973 & 1,26993 & 1,40975 & 1,07824 & 0,40327 & 2,03368 & 0,75057 \\
\hline CMIG4 & 3,13166 & 1,18599 & 2,91973 & 4,99766 & 1,95533 & 2,59944 & 1,81474 & 0,91131 & 3,37632 & 0,97113 \\
\hline CSNA3 & 4,06216 & 1,23358 & 1,26993 & 1,95533 & 2,84367 & 0,38658 & 0,31901 & 1,70771 & 3,30038 & 2,18217 \\
\hline EMBR3 & 1,14158 & 0,66608 & 1,40975 & 2,59944 & 0,38658 & 1,97924 & 1,27085 & 1,54689 & 1,41576 & 0,13378 \\
\hline GGBR4 & 0,66529 & 0,29070 & 1,07824 & 1,81474 & 0,31901 & 1,27085 & 1,13765 & 1,22575 & 0,96669 & 0,38715 \\
\hline ITSA4 & 2,02945 & 0,18684 & 0,40327 & 0,91131 & 1,70771 & 1,54689 & 1,22575 & 3,18869 & 1,03238 & 1,01533 \\
\hline $\begin{array}{l}\text { PETR4 } \\
\end{array}$ & 5,13989 & 1,77561 & 2,03368 & 3,37632 & 3,30038 & 1,41576 & 0,96669 & 1,03238 & 4,51638 & 2,64995 \\
\hline VALE5 & 3,17027 & 1,08012 & 0,75057 & 0,97113 & 2,18217 & 0,13378 & 0,38715 & 1,01533 & 2,64995 & 3,18750 \\
\hline
\end{tabular}

Source: The author 
DOI: 10.14807/ijmp.v7i1.324

Table 20: Covariance matrix of $2^{\text {nd }}$ semester of 2012

\begin{tabular}{|c|c|c|c|c|c|c|c|c|c|c|}
\hline \multicolumn{11}{|c|}{ 20 SEMESTRE 2012} \\
\hline & BBAS3 & BBDC4 & CCRO3 & CMIG4 & CSNA3 & EMBR3 & GGB & ITUB.K & PETR4 & VALE5 \\
\hline BBAS3 & 3,28933 & 2,17496 & 0,58054 & 2,36866 & 0,74883 & 0,47601 & 0,34863 & 0,51171 & 1,68187 & 0,32253 \\
\hline BBDC4 & 2,17496 & 2,80084 & 0,87850 & 2,20863 & 0,47513 & 0,41649 & 0,30763 & 0,03131 & 0,40935 & 0,67816 \\
\hline CCRO3 & 0,58054 & 0,87850 & 0,77622 & 1,93930 & 0,00769 & 0,28620 & 0,39916 & 0,37758 & 0,01264 & 0,24862 \\
\hline CMIG4 & 2,36866 & 2,20863 & 1,93930 & 12,71010 & 0,94578 & 1,41416 & 2,00817 & 2,86852 & 1,24497 & 1,50049 \\
\hline CSNA3 & 0,74883 & 0,47513 & 0,00769 & 0,94578 & 0,55198 & 0,18891 & 0,08100 & 0,23511 & 0,54562 & 0,67921 \\
\hline EMBR3 & 0,47601 & 0,41649 & 0,28620 & 1,41416 & 0,18891 & 0,36323 & 0,23326 & 0,35360 & 0,30879 & 0,12144 \\
\hline GGBR4 & 0,34863 & 0,30763 & 0,39916 & 2,00817 & 0,08100 & 0,23326 & 0,47938 & 0,63210 & 0,23014 & 0,02634 \\
\hline ITSA4 & 0,51171 & 0,03131 & 0,37758 & 2,86852 & 0,23511 & 0,35360 & 0,63210 & 1,10605 & 0,65776 & 0,07442 \\
\hline PETR4 & 1,68187 & 0,40935 & 0,01264 & 1,24497 & 0,54562 & 0,30879 & 0,23014 & 0,65776 & 1,80913 & 0,81695 \\
\hline VALE5 & 0,32253 & 0,67816 & 0,24862 & 1,50049 & 0,67921 & 0,12144 & 0,02634 & 0,07442 & 0,81695 & 3,92926 \\
\hline
\end{tabular}

Source: The author

Based on these matrices (Table 3 to 20), we can infer that the covariances are not stable over time, which would put at risk the maintenance over time of portfolios of investment according to the Markowitz model (1952). It is also important to note that these variabilities further increase in periods of crisis, when a significant increase in covariance is observed among the majority of shares in 2007 and, especially, in 2008.

There is a reasonable peak increase in the $2^{\text {nd }}$ semester of 2007, followed by a slight drop in the $1^{\text {st }}$ semester of 2008. And later, a substantially higher peak approximately, $700 \%$ higher - in the $1^{\text {st }}$ semester of 2008 , reaching the maximum covariance of the nine years studied in this work. Thus, in general, the tables present a growing instability in the $1^{\text {st }}$ semester of 2004 until the $2^{\text {nd }}$ semester of 2007, when the summit is reached in 2008. Consecutively, from the $1^{\text {st }}$ semester of 2009 until the $2^{\text {nd }}$ semester of 2012, instabilities are perceived and they still exist, although decreasing.

Another secondary result is the stable relation of CMIG4 and CCRO3 have when compared to the others. Using a simple measurement of dispersion, namely the interval of variation, calculated by the difference between the maximum and minimum in the observation dataset, we found that CMIG4 and CCRO4 had 6,29\% and $6,38 \%$ of variation respectively. As opposed to the GGB share with a very unstable variation of $45,38 \%$.

In the following chart such a conclusion can be more visually observed. 
INDEPENDENT JOURNAL OF MANAGEMENT \& PRODUCTION (IJM\&P)

http://www.ijmp.jor.br

v. 7, n. 1, January - March 2016

ISSN: 2236-269X

DOI: 10.14807/ijmp.v7i1.324

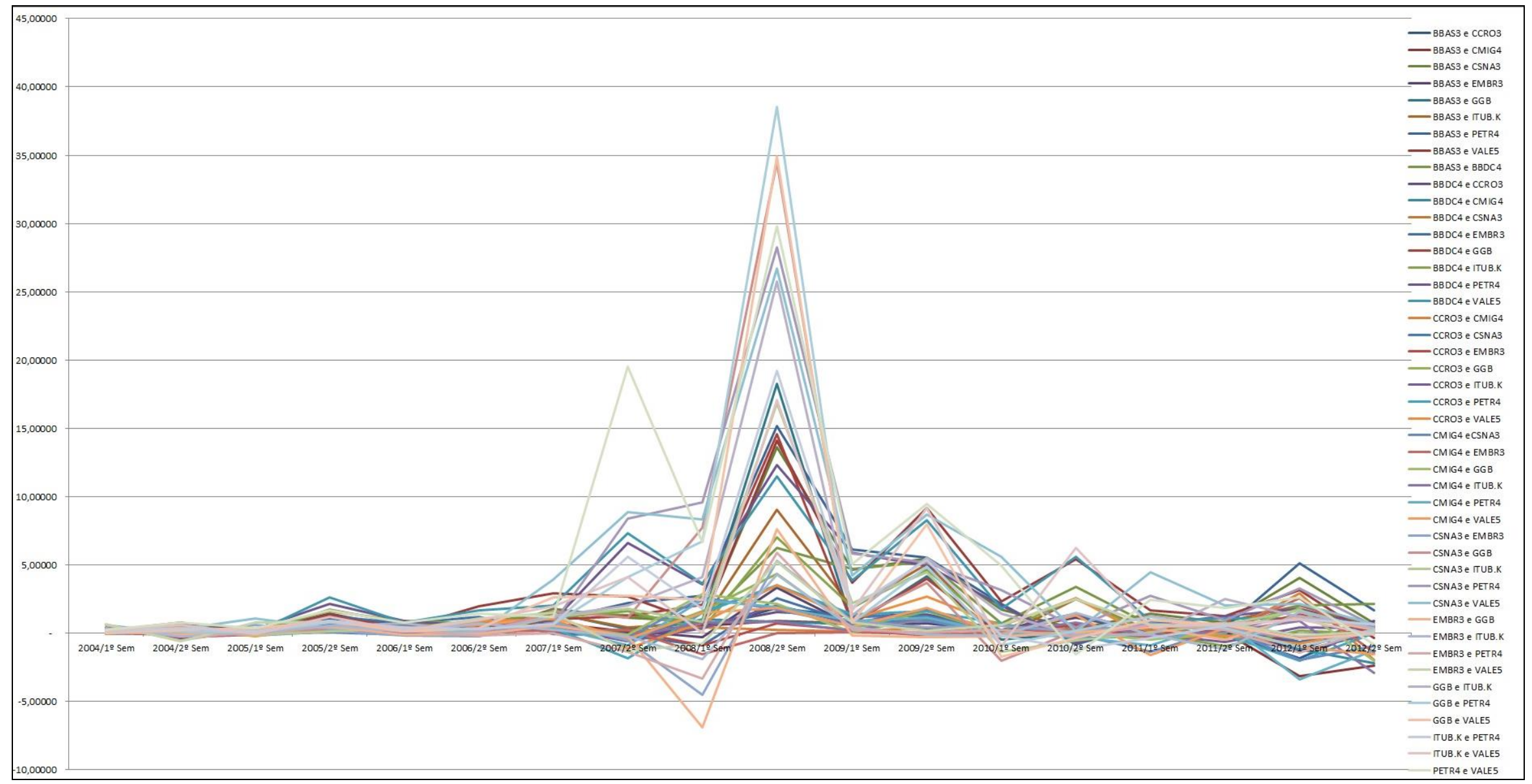

Chart 2: Behavior of covariance between shares returns over time Source: The author 
INDEPENDENT JOURNAL OF MANAGEMENT \& PRODUCTION (IJM\&P)

http://www.ijmp.jor.br

v. 7, n. 1, January - March 2016

ISSN: 2236-269X

DOI: 10.14807/ijmp.v7i1.324

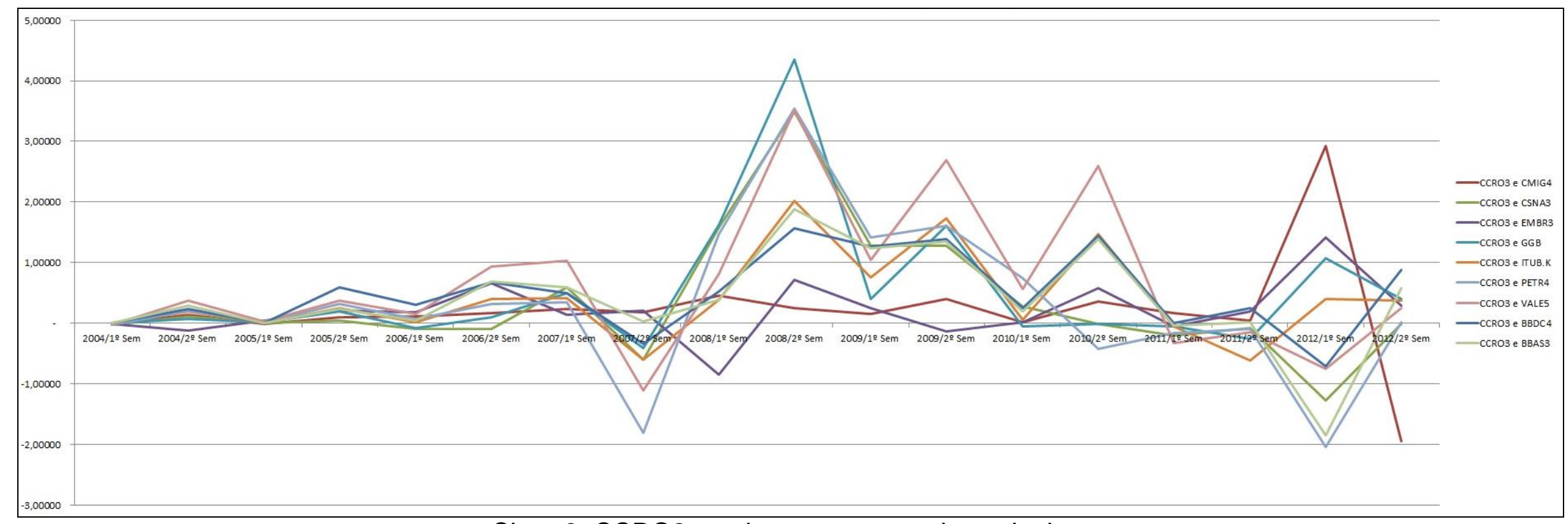

Chart 3: CCRO2 to other assets covariance in time Source: The author 
INDEPENDENT JOURNAL OF MANAGEMENT \& PRODUCTION (IJM\&P)

http://www.ijmp.jor.br

v. 7, n. 1, January - March 2016

ISSN: 2236-269X

DOI: 10.14807/ijmp.v7i1.324

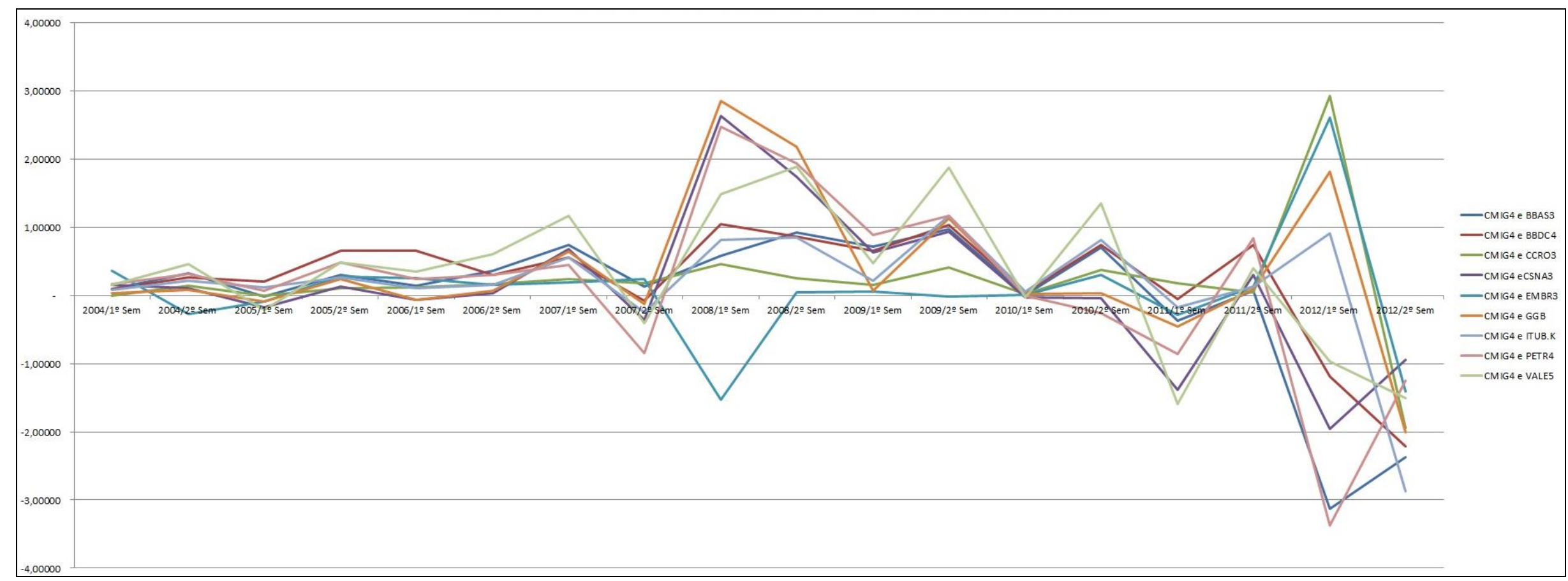

Chart 4: CMIG4 to other assets covariance in time

Source: The author 
INDEPENDENT JOURNAL OF MANAGEMENT \& PRODUCTION (IJM\&P)

http://www.ijmp.jor.br

v. 7, n. 1, January - March 2016

ISSN: 2236-269X

DOI: 10.14807/ijmp.v7i1.324

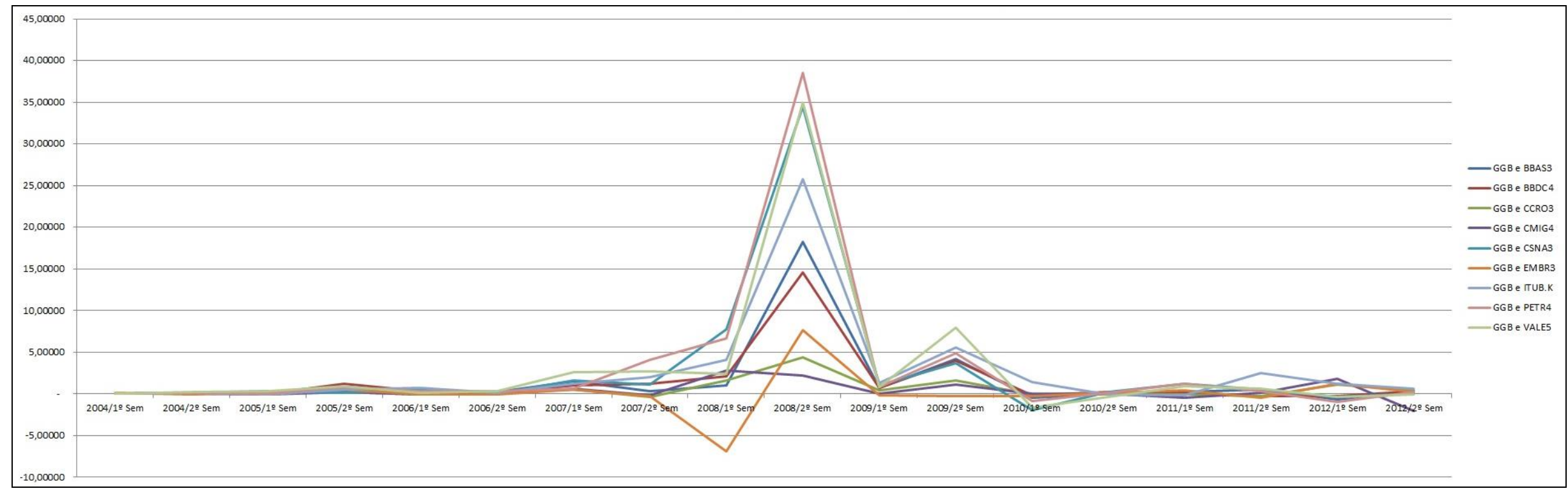

Chart 5: GGB to other assets covariance in time

Source: The author

\section{CONSTRUCTING HYPOTHETICAL PORTFOLIO}

After concluding that the instability of the covariances between the shares not only exist, but it is also significant, a more particular evaluation of these oscillations is necessary, from the construction of six hypothetical portfolios in relation to the periods of pre-crisis, crisis and post-crisis. These portfolios seek to identify the influence of covariance instability in the maintenance cost of the portfolios. 
DOI: 10.14807/ijmp.v7i1.324

We formed two species of hypothetical portfolios for each period aforementioned. Both types of portfolios do not allow short selling, that is, $\sum_{i=1}^{n} W_{i}=1$. However, one of them has as a constraint, obtaining a Sharpe index

equivalent to $15 \%$ for its formation. The other portfolio genre should present constant asset weight over time, and it starts with a distribution that generates a Sharpe index also equivalent to $15 \%$. In the following we present the data to obtain each portfolio returns, standard deviations and covariance matrices - as well as the construction of portfolios with the respective weights of each asset.

\subsection{Hypothetical portfolio pre-crisis: January/2004 to June/2007}

Table 21: Data for individual assets in the pre-crisis period

\begin{tabular}{|c|c|c|}
\hline Assets & $\boldsymbol{\mu}$ & $\boldsymbol{\sigma}$ \\
\hline BBAS3 & $253,20 \%$ & $365,21 \%$ \\
\hline BBDC4 & $270,12 \%$ & $499,17 \%$ \\
\hline CCRO3 & $416,51 \%$ & $143,23 \%$ \\
\hline CMIG4 & $129,92 \%$ & $152,94 \%$ \\
\hline CSNA3 & $150,99 \%$ & $179,99 \%$ \\
\hline EMBR3 & $20,23 \%$ & $282,37 \%$ \\
\hline GGBR4 & $410,43 \%$ & $248,11 \%$ \\
\hline ITUB.K & $352,20 \%$ & $339,76 \%$ \\
\hline PETR4 & $160,81 \%$ & $422,94 \%$ \\
\hline VALE5 & $187,09 \%$ & $538,25 \%$ \\
\hline \multicolumn{3}{|c|}{ Source The author } \\
\hline
\end{tabular}

Table 22: Covariance between assets in the pre-crisis period

\begin{tabular}{|l|c|c|c|c|c|c|c|c|c|c|}
\hline & BBAS3 & BBDC4 & CCRO3 & CMIG4 & CSNA3 & EMBR3 & GGBR4 & ITUB.K & PETR4 & VALE5 \\
\hline BBAS3 & 13.34 & 17.44 & 5.03 & 5.26 & 5.82 & 8.82 & 8.77 & 12.02 & 14.54 & 18.95 \\
\hline BBDC4 & 17.44 & 24.92 & 6.73 & 7.41 & 7.29 & 11.78 & 11.63 & 16.65 & 20.62 & 24.60 \\
\hline CCRO3 & 5.03 & 6.73 & 2.05 & 2.03 & 2.20 & 3.36 & 3.32 & 4.67 & 5.53 & 7.39 \\
\hline CMIG4 & 5.26 & 7.41 & 2.03 & 2.34 & 2.18 & 3.32 & 3.47 & 4.99 & 6.21 & 7.50 \\
\hline CSNA3 & 5.82 & 7.29 & 2.20 & 2.18 & 3.24 & 4.14 & 4.06 & 5.18 & 6.21 & 8.73 \\
\hline EMBR3 & 8.82 & 11.78 & 3.36 & 3.32 & 4.14 & 7.97 & 6.03 & 8.02 & 9.94 & 12.44 \\
\hline GGBR4 & 8.77 & 11.63 & 3.32 & 3.47 & 4.06 & 6.03 & 6.16 & 8.12 & 9.91 & 12.35 \\
\hline ITUB.K & 12.02 & 16.65 & 4.67 & 4.99 & 5.18 & 8.02 & 8.12 & 11.54 & 13.86 & 17.15 \\
\hline PETR4 & 14.54 & 20.62 & 5.53 & 6.21 & 6.21 & 9.94 & 9.91 & 13.86 & 17.89 & 20.21 \\
\hline VALE5 & 18.95 & 24.60 & 7.39 & 7.50 & 8.73 & 12.44 & 12.35 & 17.15 & 20.21 & 28.97 \\
\hline
\end{tabular}


DOI: 10.14807/ijmp.v7i1.324

Table 23: Assets portfolios in the pre-crisis period

\begin{tabular}{|c|c|c|}
\hline & IS $=15 \%$ & Constant weights \\
\hline Assets & \multicolumn{2}{|c|}{ Wi } \\
\hline BBAS3 & $0,00 \%$ & $0,00 \%$ \\
\hline BBDC4 & $0,00 \%$ & $0,00 \%$ \\
\hline CCRO3 & $0,00 \%$ & $0,00 \%$ \\
\hline CMIG4 & $0,00 \%$ & $0,00 \%$ \\
\hline CSNA3 & $0,00 \%$ & $0,00 \%$ \\
\hline EMBR3 & $83,83 \%$ & $83,83 \%$ \\
\hline GGBR4 & $0,00 \%$ & $0,00 \%$ \\
\hline ITUB.K & $0,00 \%$ & $0,00 \%$ \\
\hline PETR4 & $4,14 \%$ & $4,14 \%$ \\
\hline VALE5 & $12,03 \%$ & $12,03 \%$ \\
\hline Iwi & $100,00 \%$ & $100,00 \%$ \\
\hline Hp & $46,12 \%$ & $46,12 \%$ \\
\hline op & $307,46 \%$ & $307,46 \%$ \\
\hline IS & $15,00 \%$ & $15,00 \%$ \\
\hline & Source: The author \\
\hline
\end{tabular}

\subsection{Crisis hypothetical portfolio: July/2007 to June/2009}

Table 24: Data for individual assets in the pre-crisis period

\begin{tabular}{|c|c|c|}
\hline Assets & $\boldsymbol{\mu}$ & $\boldsymbol{\sigma}$ \\
\hline BBAS3 & $20,72 \%$ & $387,24 \%$ \\
\hline BBDC4 & $25,78 \%$ & $311,41 \%$ \\
\hline CCRO3 & $22,57 \%$ & $101,15 \%$ \\
\hline CMIG4 & $5,70 \%$ & $74,18 \%$ \\
\hline CSNA3 & $86,07 \%$ & $498,03 \%$ \\
\hline EMBR3 & $-56,67 \%$ & $432,06 \%$ \\
\hline GGBR4 & $35,35 \%$ & $511,16 \%$ \\
\hline ITUB.K & $33,09 \%$ & $344,08 \%$ \\
\hline PETR4 & $51,00 \%$ & $570,36 \%$ \\
\hline VALE5 & $22,14 \%$ & $701,37 \%$ \\
\hline
\end{tabular}

Source: The author 
INDEPENDENT JOURNAL OF MANAGEMENT \& PRODUCTION (IJM\&P)

http://www.ijmp.jor.br

v. 7, n. 1, January - March 2016

ISSN: 2236-269X

DOI: 10.14807/ijmp.v7i1.324

Table 25: Covariance between assets in the pre-crisis period

\begin{tabular}{|l|c|c|c|c|c|c|c|c|c|c|c|}
\hline & BBAS3 & BBDC4 & CCRO3 & CMIG4 & CSNA3 & EMBR3 & GGBR4 & ITUB.K & PETR4 & VALE5 \\
\hline BBAS3 & 15,00 & 11,22 & 3,19 & 1,06 & 12,46 & 7,31 & 10,07 & 10,40 & 14,87 & 20,75 \\
\hline BBDC4 & 11,22 & 9,70 & 2,32 & 0,87 & 11,38 & 6,17 & 9,52 & 8,65 & 13,50 & 18,82 \\
\hline CCRO3 & 3,19 & 2,32 & 1,02 & 0,48 & 2,40 & 0,61 & 1,89 & 2,04 & 2,35 & 3,33 \\
\hline CMIG4 & 1,06 & 0,87 & 0,48 & 0,55 & 0,89 & $-0,76$ & 1,11 & 0,72 & 0,71 & 0,41 \\
\hline CSNA3 & 12,46 & 11,38 & 2,40 & 0,89 & 24,80 & 1,15 & 16,62 & 11,14 & 26,07 & 24,47 \\
\hline EMBR3 & 7,31 & 6,17 & 0,61 & $-0,76$ & 1,15 & 18,67 & 7,11 & 6,63 & 4,01 & 19,90 \\
\hline GGBR4 & 10,07 & 9,52 & 1,89 & 1,11 & 16,62 & 7,11 & 26,13 & 15,20 & 18,12 & 21,90 \\
\hline ITUB.K & 10,40 & 8,65 & 2,04 & 0,72 & 11,14 & 6,63 & 15,20 & 11,84 & 13,22 & 18,07 \\
\hline PETR4 & 14,87 & 13,50 & 2,35 & 0,71 & 26,07 & 4,01 & 18,12 & 13,22 & 32,53 & 31,19 \\
\hline VALE5 & 20,75 & 18,82 & 3,33 & 0,41 & 24,47 & 19,90 & 21,90 & 18,07 & 31,19 & 49,19 \\
\hline
\end{tabular}

Table 26: Assets portfolios in the crisis period

\begin{tabular}{|c|c|c|}
\hline & IS $=\mathbf{1 5} \%$ & Constant weights \\
\hline Assets & \multicolumn{2}{|c|}{ Wi } \\
\hline BBAS3 & $0,00 \%$ & $0,00 \%$ \\
\hline BBDC4 & $3,46 \%$ & $0,00 \%$ \\
\hline CCRO3 & $20,09 \%$ & $0,00 \%$ \\
\hline CMIG4 & $14,89 \%$ & $0,00 \%$ \\
\hline CSNA3 & $28,16 \%$ & $0,00 \%$ \\
\hline EMBR3 & $0,00 \%$ & $83,83 \%$ \\
\hline GGBR4 & $9,22 \%$ & $0,00 \%$ \\
\hline ITUB.K & $13,46 \%$ & $0,00 \%$ \\
\hline PETR4 & $10,72 \%$ & $4,14 \%$ \\
\hline VALE5 & $0,00 \%$ & $12,03 \%$ \\
\hline Iwi & $100,00 \%$ & $100,00 \%$ \\
\hline Mp & $43,69 \%$ & $-42,73 \%$ \\
\hline op & $291,27 \%$ & $429,97 \%$ \\
\hline IS & $15,00 \%$ & $-9,94 \%$ \\
\hline
\end{tabular}

Source: The author

\subsection{Hypothetical portfolio post-crisis: July/2009 to December/2012}

Table 27: Data for individual assets in the post-crisis period

\begin{tabular}{|c|c|c|}
\hline Assets & $\boldsymbol{\mu}$ & $\boldsymbol{\sigma}$ \\
\hline BBAS3 & $-4,74 \%$ & $214,79 \%$ \\
\hline BBDC4 & $-24,48 \%$ & $225,07 \%$ \\
\hline CCRO3 & $-118,61 \%$ & $296,50 \%$ \\
\hline CMIG4 & $-47,94 \%$ & $358,54 \%$ \\
\hline CSNA3 & $50,82 \%$ & $527,22 \%$ \\
\hline EMBR3 & $-56,65 \%$ & $174,90 \%$ \\
\hline GGBR4 & $47,29 \%$ & $289,20 \%$ \\
\hline ITUB.K & $31,06 \%$ & $285,78 \%$ \\
\hline PETR4 & $42,40 \%$ & $360,04 \%$ \\
\hline VALE5 & $-8,45 \%$ & $300,85 \%$ \\
\hline \multicolumn{2}{|c|}{ Source: The author } \\
\hline
\end{tabular}


INDEPENDENT JOURNAL OF MANAGEMENT \& PRODUCTION (IJM\&P)

http://www.ijmp.jor.br

v. 7, n. 1, January - March 2016

ISSN: 2236-269X

DOI: 10.14807/ijmp.v7i1.324

Table 28: Covariance between assets in the post-crisis period

\begin{tabular}{|l|c|c|c|c|c|c|c|c|c|c|}
\hline & BBAS3 & BBDC4 & CCRO3 & CMIG4 & CSNA3 & EMBR3 & GGBR4 & ITUB.K & PETR4 & VAE5 \\
\hline BBAS3 & 4,61 & 1,23 & $-2,49$ & $-3,74$ & 7,24 & $-0,54$ & 2,65 & 3,65 & 3,70 & 4,23 \\
\hline BBDC4 & 1,23 & 5,07 & 4,64 & 3,48 & $-4,36$ & 2,61 & $-2,67$ & $-2,06$ & $-2,73$ & 0,82 \\
\hline CCRO3 & $-2,49$ & 4,64 & 8,79 & 8,23 & $-12,63$ & 4,07 & $-5,79$ & $-5,54$ & $-6,77$ & $-2,95$ \\
\hline CMIG4 & $-3,74$ & 3,48 & 8,23 & 12,86 & $-13,52$ & 4,38 & $-6,81$ & $-5,61$ & $-7,55$ & $-3,43$ \\
\hline CSNA3 & 7,24 & $-4,36$ & $-12,63$ & $-13,52$ & 27,80 & $-4,83$ & 13,09 & 10,35 & 15,87 & 8,90 \\
\hline EMBR3 & $-0,54$ & 2,61 & 4,07 & 4,38 & $-4,83$ & 3,06 & $-2,58$ & $-1,42$ & $-3,26$ & 0,25 \\
\hline GGBR4 & 2,65 & $-2,67$ & $-5,79$ & $-6,81$ & 13,09 & $-2,58$ & 8,36 & 5,61 & 8,19 & 2,69 \\
\hline ITUB.K & 3,65 & $-2,06$ & $-5,54$ & $-5,61$ & 10,35 & $-1,42$ & 5,61 & 8,17 & 4,75 & 5,10 \\
\hline PETR4 & 3,70 & $-2,73$ & $-6,77$ & $-7,55$ & 15,87 & $-3,26$ & 8,19 & 4,75 & 12,96 & 4,16 \\
\hline VALE5 & 4,23 & 0,82 & $-2,95$ & $-3,43$ & 8,90 & 0,25 & 2,69 & 5,10 & 4,16 & 9,05 \\
\hline
\end{tabular}

Source: The author

Table 29: Assets portfolios in the post-crisis period

\begin{tabular}{|c|c|c|}
\hline & IS = 15\% & Constant weights \\
\hline Assets & \multicolumn{2}{|c|}{ Wi } \\
\hline BBAS3 & $0.00 \%$ & $0.00 \%$ \\
\hline BBDC4 & $0.00 \%$ & $0.00 \%$ \\
\hline CCRO3 & $0.00 \%$ & $0.00 \%$ \\
\hline CMIG4 & $0.00 \%$ & $0.00 \%$ \\
\hline CSNA3 & $0.34 \%$ & $0.00 \%$ \\
\hline EMBR3 & $0.00 \%$ & $83.83 \%$ \\
\hline GGBR4 & $39.48 \%$ & $0.00 \%$ \\
\hline ITUB.K & $29.28 \%$ & $0.00 \%$ \\
\hline PETR4 & $30.90 \%$ & $4.14 \%$ \\
\hline VALE5 & $0.00 \%$ & $12.03 \%$ \\
\hline Iwi & $100.00 \%$ & $100.00 \%$ \\
\hline Hp & $41.04 \%$ & $-46.75 \%$ \\
\hline op & $273.60 \%$ & $147.23 \%$ \\
\hline IS & $15.00 \%$ & $-31.75 \%$ \\
\hline
\end{tabular}

Source: The author

Where:

- $\mu=$ return of a given asset in the corresponding period;

- $\sigma=$ standard deviation of a given asset in the corresponding period;

- SI = Sharpe index;

- $\Sigma w i=$ total sum of assets weights;

- $\quad \mu_{p}=$ total expected return of the portfolio;

- $\sigma_{p}=$ total standard deviation of a portfolio. 
DOI: 10.14807/ijmp.v7i1.324

The preferences of return and risk of an investor are one of the most important factors to be considered in assembling portfolios, as seen in the theory. From this analysis it is evident that in order to maintain such preferences, in the case of a rate of beyond $15 \%$ of return for each additional unit of risk, it is necessary to change periodically the assets weights in the hypothetical portfolio. If the investor does not recalculate the assets weights of their portfolio as shown by the type of hypothetical portfolio of constant weights, their preference regarding return and risk expected is not met over time. Nevertheless, if the investor wishes to rescue the application in times of crisis or post-crisis, they will have a loss of $-42.73 \%$ or $-46.75 \%$, respectively, of the initial investment made in January 2004 (pre-crisis). The behavior of returns and risks during the studied period can be verified according to the following charts.

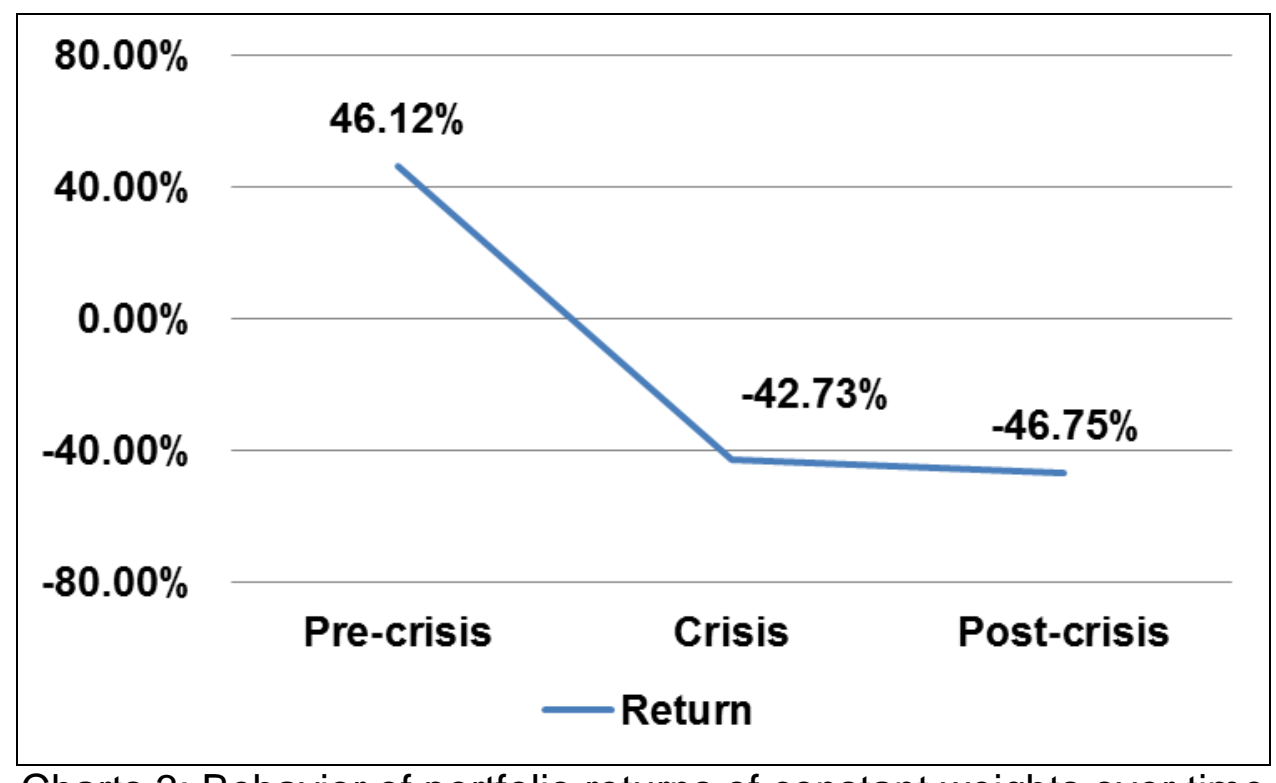

Charts 3: Behavior of portfolio returns of constant weights over time Source: The author 
DOI: 10.14807/ijmp.v7i1.324

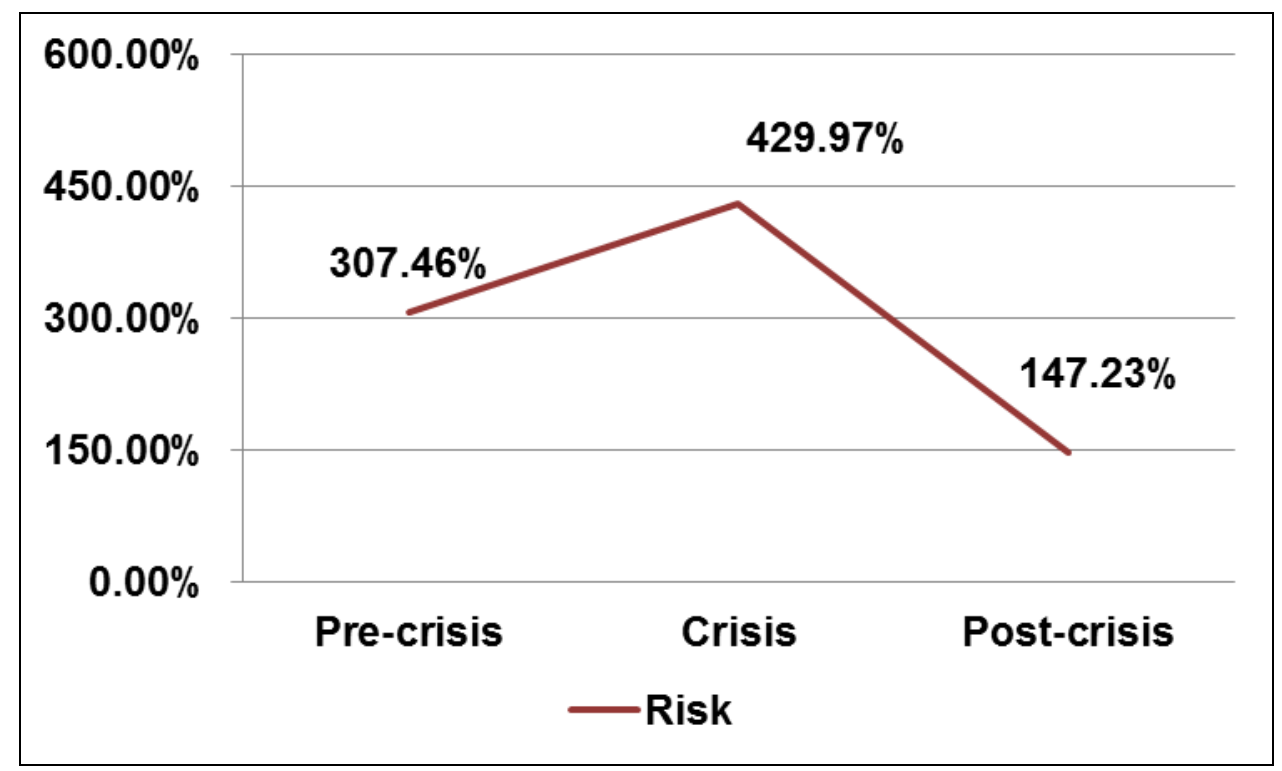

Chart 4: Behavior of portfolios risk of constant weights over time Source: The author

It is interesting to note that the Petrobras share remains in the three hypothetical portfolios whose premise is a constant Sharpe index equal to $15 \%$.

\section{CONCLUSION}

As we could observe in the theoretical foundation, the correlation index is a measure resulting from the ratio of the covariance and standard deviations of the analyzed elements. Thus, keeping everything else constant, as their covariance changes, the correlation between them also changes. Consequently, knowing that the risk-return curve has its curvature defined by the correlation itself, this curvature will depend on the changes undergone by the covariance statistics.

According to the charts we can clearly observe that the covariances, originated from the returns of the companies, are not stable over time, and in times of crisis they vary even more. These changes reflect in the risk-return curves, so to modify the possible sets of portfolios to be assembled and, therefore, the asset allocation within these portfolios. This observation is properly shown on chart 1 .

This means that the portfolios assembly according to the Markowitz model works only for a period - which is lower or higher in accordance to the economic turmoil which oscillates the covariance between the returns of the companies - the portfolio must be constantly recomposed. In other words, given a specific set of shares, the holding of each share must be periodically recalculated, as demonstrated, in order to always adequate the preferences of a particular investor. 
DOI: 10.14807/ijmp.v7i1.324

As demonstrated in this research, share weights will change more in the portfolio in times of crisis, in which the covariances between companies change substantially.

Periodically recalculate the holding of each asset of the investment portfolio is a possible solution to the instability problem of covariance over time. However, it might increase the maintenance costs of portfolio which will be as costly as larger are the covariance volatilities. Another interesting solution to be explored in future studies is by identifying portfolios according to the degree of stability of their covariances which could be measured most precisely with the aid of a statistical hypothesis test.

\section{REFERENCES}

ALMEIDA, N.; SILVA, R. F.; RIBEIRO, K.(2010) Aplicação do modelo de Markowitz na seleção de carteiras eficientes: uma análise de cenários no mercado de capitais brasileiro. XIII Seminários em administração, Uberlândia. Setembro.

HIEDA, A.; ODA, A. L. (1998) Um estudo sobre a utilização de dados históricos no modelo de Markowitz aplicado a Bolsa de Valores de São Paulo. In:

Seminários de Administração, 3, Out. 1998, São Paulo. Anais do III SEMEAD. São Paulo: Faculdade de Economia, Administração e Contabilidade da USP.

BODIE, Z.; MARCUS, A. J.; KANE, A. (2010) Investimentos. New York: Artmed.

DANTHINE, J.; DONALDSON, J. B. (2005) Intermediate financial theory.

California: Elsevier.

LUENBERGER, D. G. (1997) Investment Science. USA: Oxford University Press. p. 137-172.

MARKOWITZ, Harry M. (1952) Portfólio selection. Journal of Finance, v. 7, n. 1, p. 77-91. Mar.

PENTEADO, M. A.; FAMÁ, R. (2002) Será que o beta que temos é o beta que queremos?. Cadernos de pesquisas em administração, São Paulo, v. 09, n. 3, julho/setembro.

SHARPE, William F.; ALEXANDER, GORDON J.; BAILEY, JEFFERY V. (1995) Investments. NewJersey: Prentice Hall. 